\title{
Physical properties of seven binary and higher-order multiple OB systems ${ }^{\star}$
}

\author{
Pavel Mayer ${ }^{1}$, Petr Harmanec ${ }^{1}$, Rolf Chini ${ }^{2,3}$, Anita Nasseri ${ }^{2}$, Jana A. Nemravová ${ }^{1}$, Horst Drechsel ${ }^{4}$, \\ Rodrigo Catalan-Hurtado ${ }^{5}$, Brad N. Barlow ${ }^{5}$, Yves Frémat ${ }^{6}$, and Lenka Kotková ${ }^{7}$ \\ 1 Astronomical Institute of the Charles University, Faculty of Mathematics and Physics, V Holešovičkách 2, 18000 Praha 8, \\ Czech Republic \\ e-mail: mayer@cesnet.cz \\ 2 Astronomisches Institut, Ruhr-Universität Bochum, Universitätsstr. 150, 44801 Bochum, Germany \\ 3 Instituto de Astronomía, Universidad Católica del Norte, Avenida Angamos 0610, Casilla 1280, Antofagasta, Chile \\ ${ }^{4}$ Dr. Karl Remeis-Observatory \& ECAP, Astronomical Institute, Friedrich-Alexander-University Erlangen-Nuremberg, \\ Sternwartstr. 7, 96049 Bamberg, Germany \\ 5 Department of Physics, High Point University, One University Way, High Point, NC 27268, USA \\ ${ }^{6}$ Royal Observatory of Belgium, Avenue circulaire 3, 1180 Brussels, Belgium \\ 7 Astronomical Institute, Academy of Sciences of the Czech Republic, 25165 Ondřejov, Czech Republic
}

Received 4 May 2016 / Accepted 14 December 2016

\begin{abstract}
Analyses of multi-epoch, high-resolution $(R \sim 50000)$ optical spectra of seven early-type systems provided various important new insights with respect to their multiplicity. First determinations of orbital periods were made for HD $92206 \mathrm{C}$ (2.022), HD 112244

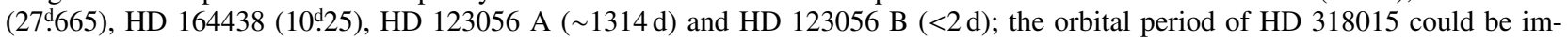
proved (23.445975). Concerning multiplicity, a third component was discovered for HD $92206 \mathrm{C}$ by means of He I line profiles. For HD 93146 A, which was hitherto assumed to be SB1, lines of a secondary component could be discerned. HD 123056 turns out to be a multiple system consisting of a high-mass component $\mathrm{A}(\approx \mathrm{O} 8.5)$ displaying a broad He II $5411 \AA$ feature with variable radial velocity, and of an inner pair B ( $\approx$ B0) with double He I lines. The binary HD 164816 was revisited and some of its system parameters were improved. In particular, we determined its systemic velocity to be $-7 \mathrm{~km} \mathrm{~s}^{-1}$, which coincides with the radial velocity of the cluster NGC 6530. This fact, together with its distance, suggests the cluster membership of HD 164816. The OB system HD 318015 (V1082 Sco) belongs to the rare class of eclipsing binaries with a supergiant primary (B0.5/0.7). Our combined orbital and light-curve analysis suggests that the secondary resembles an O9.5 III star. Our results for a limited sample corroborate the findings that many $\mathrm{O}$ stars are actually massive multiple systems.
\end{abstract}

Key words. binaries: spectroscopic - stars: massive - stars: fundamental parameters - stars: general

\section{Introduction}

Duplicity or even multiplicity of $\mathrm{O}$ stars is a topic that has attracted attention from various aspects, mainly related to the origin and evolution of massive stars. While Garmany et al. (1980), for example, list only $40 \mathrm{O}$ galactic-type binaries with known orbits in the survey of the binary frequency of $\mathrm{O}$ stars, this fraction has been growing steadily. Mason et al. (1998) obtained speckle interferometry of $227 \mathrm{O}$ stars brighter than $V=8.0$ and compiled information about their spectroscopic duplicity or multiplicity from the literature. They concluded that the duplicity is higher for stars in clusters and associations (72\%) than for the

\footnotetext{
^ Based on spectra from observations made with ESO telescopes at La Silla and Paranal Observatories under programs 065.1-0526(A), 073.C-0337(A), 074.D-0300(A), 075.D-0103(A), 077.D-0705(A), 077.B-0348(A), 079.D-0564(A), 081.D-2008(A), 083.D-0589(A), 085.D-0262(A), 086.D-0997(A)， 087.C-0012(A), 087.D-0946(A), 089.C-0415(A), 089.D-0097(B), 089.D-0975(A), 090.C-0280(A), 091.D-0145(A), 095.A-9032(A), and 178.D-0361(E), program TYCHO $\mathrm{P} 2$, on the BESO spectra and spectra from CTIO, and on the HIPPARCOS $H_{\mathrm{p}}$ and ASAS3 $V$ photometry.
}

field stars (19\%); the lowest being for runaway stars (8\%). They also noted that many $\mathrm{O}$ stars are actually triple systems. In continuation of this study (Mason et al. 2009), they further strengthened the multiplicity among clusters and associations. Sana et al. (2008) re-investigated the duplicity of O stars in the NGC 6231 cluster and concluded that more than $63 \%$ of $\mathrm{O}$ stars must be binaries. They pointed out that this observational fact represents an important constraint for the theories of early evolution of $\mathrm{O}$ stars. Chini et al. (2012) studied a sample of $248 \mathrm{O}$ and 581 mostly southern stars and concluded that more than $82 \%$ of stars more massive than $16 M_{\odot}$ are close binary systems and that this fraction decreases to $20 \%$ for $3 M_{\odot}$ stars. They also found differences in the binary fractions of $\mathrm{O}$ stars in different environments: $72 \pm 13 \%$ in clusters, $73 \pm 8 \%$ in associations, $69 \pm 11 \%$ among runaway stars and $43 \pm 13 \%$ for the field stars. The fact that duplicity must be a key factor affecting the evolution of high-mass binaries was discussed in detail by Sana et al. (2012). In several recent studies the binary nature of many O-type stars was found or suspected. Sota et al. (2014) presented a survey of 448 Galactic $\mathrm{O}$ stars from both hemispheres, based on high signalto-noise ratio $(\mathrm{S} / \mathrm{N}) R \sim 2500$ spectra, which is complete to 
Table 1. Basic information about the spectra used.

\begin{tabular}{rccrc}
\hline \hline Spectrograph & Abbreviation & Spectral range $(\AA)$ & Resolution & $S / N$ range \\
\hline FEROS & F plus T & $3600-9100$ & 48000 & $63-311$ \\
HARPS & H & $3800-6912$ & 115000 & $84-106$ \\
BESO & B & $3800-8000$ & 48000 & $26-196$ \\
CTIO & C & $4500-8900$ & 28000 & $83-177$ \\
\hline
\end{tabular}

$B=8$ m 0 ; further 142 stars were listed by Maíz Apellániz et al. (2016). Combining results from a multi-epoch, high-resolution spectroscopic survey, OWN (Barbá et al. 2010), with additional information on spectroscopic and visual binaries from the literature, the authors conclude that only a very small fraction of stars with masses above 15-20 $M_{\odot}$ are born as single systems.

In the ongoing multi-epoch survey started in 2009 (Chini et al. 2012), which meanwhile comprises more than 2000 high-resolution spectra, a large number of hitherto unknown spectroscopic binaries was found. An example of the study where the spectra of this survey were analysed in detail is HD 152246, discovered as a binary by Thackeray et al. (1973). The analysis of its double-lined spectra yielded a significant difference of the orbital periods for both components. It suggests that HD 152246 is at least a hierarchical triple system consisting of an inner pair $\mathrm{Ba} / \mathrm{Bb}$ with approximately 17 and $6 M_{\odot}$, respectively, and an outer component A with a mass of approximately $20 M_{\odot}$ (Nasseri et al. 2014).

There are, of course, other studies of individual binaries where the orbit of a third body is measured interferometrically (Mayer et al. 2014) and of members of associations (Kiminki et al. 2007; Kiminki \& Kobulnicky 2012; Kobulnicky et al. 2014; Sana et al. 2011, 2013).

For more details on the subject, we refer readers to a study by Aldoretta et al. (2015), which represents a very good overview of the current status of duplicity of $\mathrm{O}$ stars and also gives a number of relevant references. The authors identified new binaries with the Hubble Space Telescope fine guidance sensor and carried out a very extended literature search that allowed them to provide an estimate of the period distribution among $\mathrm{O}$ type binaries. They concluded that it is approximately flat and discussed several selection effects and the need for additional data. However, their complete list contains only 83 spectroscopic and eclipsing binaries with known orbital period.

The present paper brings into focus seven early-type systems, for which we analysed between 18 and 98 multi-epoch spectra, obtained mainly with the BESO and FEROS spectrographs. In addition to new orbital periods, our study reveals higher multiplicities in some of the systems. Especially important in this context are systems with a supergiant component (HD 318015, HD 112244), which are still rare. Reliable determination of their basic physical properties provides a sensitive test for the models of stellar structure and evolution, especially those that also include mass loss and/or rotation. We admit that our selection of objects was mainly guided by the above-mentioned practical considerations. It is clear, however (cf. Aldoretta et al. 2015), that our contribution is not as modest as it might appear at first sight. So far, the knowledge of duplicity and/or multiplicity of OB stars has been mainly documented on statistical grounds while the orbital solutions are still missing in the majority of cases. Zinnecker \& Yorke (2007) and Sana \& Evans (2011), for instance, pointed out that their knowledge is important for the understanding of the binary origin and evolution.

\section{Data}

The data comprise two categories; those that were secured by our own observations and those downloaded from the ESO archive to complement the phase coverage of the individual systems.

\subsection{Spectrographs}

The majority of spectra were secured with the echelle spectrograph BESO $(R \sim 48000$, Fuhrmann et al. 2011) of the Universitäts-Sternwarte Bochum, which is fibre-fed to the $1.5 \mathrm{~m}$ Hexapod telescope at Cerro Armazones, Chile; the corresponding spectra are denoted as "B". Further spectra were downloaded from the ESO archive. Those ESO spectra that were secured with the echelle spectrograph FEROS $(R \sim 48000$ Kaufer et al. 1997 ) attached to the MPI 2.2 m telescope are labelled "F", the spectra from HARPS (Mayor et al. 2003) mounted at the $3.6 \mathrm{~m}$ ESO telescope are labelled "H". Some of the FEROS spectra were obtained in the framework of the observational program "Tycho Brahe" allocated to Czech astronomers; these spectra are denoted as "T". In the case of V1082 Sco = HD 318015, we also used the spectra obtained at CTIO. The CTIO spectra were secured with the echelle spectrograph CHIRON $(R \sim 28000$, Tokovinin et al. 2013) attached to the $1.5 \mathrm{~m}$ telescope and are labelled "C". The initial reductions (bias subtraction, flatfielding and wavelength calibration) were carried out using the standard pipelines for individual instruments. Rectification of the spectra and smaller cosmics and flaw-removal were carried out interactively using the program SPEFO (Horn et al. 1996; Škoda 1996), the latest version developed by the late Mr. J. Krpata. This program allows the user to define the continuum points on the computer screen for one well-exposed spectrum, through which the continuum is smoothly defined by Hermite polynomials. The user can see the fit and is able to modify it when necessary. When proceeding to the text spectrum, all previously chosen rectification points are copied to it and the user can modify their positions if necessary, depending on the noise and radial-velocity (RV) shifts. We also carefully compared the rectification of each spectrum with the first (template) spectrum to be sure about uniformity of the rectification. Similarly, the removal of cosmics can be carried out interactively on the computer screen, the deleted pixels being replaced by a spline interpolation. Basic information about all spectra is in Table 1.

\subsection{Radial-velocity and orbit determination}

Depending on the character of spectra of individual objects, we alternatively applied three different techniques of RV measurements:

1. Fit the line profiles by one, two or three Gaussian profiles.

2. Interactive comparison of the direct and flipped line profiles displayed on the computer screen using the program SPEFO.

3. Two-dimensional (2D) cross-correlation using a Fortran implementation of the TODCOR (Zucker \& Mazeh 1994) called 
P. Mayer et al.: Seven early-type binary and multiple systems

Table 2. RVs of HD 92206 C measured via Gaussian fits of the line profiles.

\begin{tabular}{ccrrrrrrl}
\hline \hline RJD & \multirow{2}{*}{ Phase } & \multicolumn{3}{c}{5411 primary } & \multicolumn{3}{c}{5876 secondary } & \multirow{2}{*}{ Spg. } \\
& & RV & error & O-C & RV & error & O-C & \\
\hline 54209.5862 & 0.2537 & -213.5 & 8 & 5.7 & 278 & 7 & -12.3 & $\mathrm{~F}$ \\
54209.5979 & 0.2595 & -212.5 & 8 & 6.4 & 277 & 7 & -12.8 & $\mathrm{~F}$ \\
54246.5539 & 0.5313 & 19.5 & 11 & -11.4 & & & & $\mathrm{~F}$ \\
54246.5627 & 0.5357 & 32.0 & 8 & -4.5 & & & & $\mathrm{~F}$ \\
54247.5640 & 0.0321 & -52.5 & 17 & -0.6 & & & & $\mathrm{~F}$ \\
54600.5388 & 0.5550 & 56.5 & 17 & -4.4 & -144 & 14 & -47.4 & $\mathrm{~F}$ \\
54601.5493 & 0.0546 & -95.0 & 14 & -14.7 & 138 & 20 & 39.6 & $\mathrm{~F}$ \\
55016.4880 & 0.2180 & -221.0 & 11 & -6.4 & 304 & 17 & 19.5 & $\mathrm{~B}$ \\
55251.7260 & 0.5283 & 30.5 & 8 & 3.4 & & & & $\mathrm{~B}$ \\
55252.7361 & 0.0276 & -32.0 & 14 & 14.1 & & & & $\mathrm{~B}$ \\
55255.7089 & 0.4976 & -13.0 & 8 & 0.1 & & & & $\mathrm{~B}$ \\
55256.7212 & 0.9981 & 3.0 & 14 & 10.5 & & & & $\mathrm{~B}$ \\
55289.6709 & 0.2896 & -208.5 & 11 & 4.3 & 270 & 7 & -11.4 & $\mathrm{~B}$ \\
55294.7294 & 0.7908 & 206.5 & 11 & 14.0 & -300 & 11 & -21.6 & $\mathrm{~B}$ \\
56067.5121 & 0.8802 & 137.5 & 8 & 4.4 & -171 & 5 & 25.3 & $\mathrm{~F}$ \\
56687.8253 & 0.6038 & 120.0 & 8 & 2.9 & -162 & 14 & 12.2 & $\mathrm{~B}$ \\
56730.6903 & 0.7978 & 190.0 & 8 & 0.0 & -266 & 7 & 8.9 & $\mathrm{~B}$ \\
56740.6454 & 0.7201 & 206.0 & 8 & 10.4 & & & & $\mathrm{~B}$ \\
\hline
\end{tabular}

Notes. All RVs are in $\mathrm{km} \mathrm{s}^{-1}$. The last column "Spg." denotes the spectrograph.

asTODCOR, developed by one of the present authors (YF) and first applied to the study of AU Mon (Desmet et al. 2010).

Whenever using the first two techniques, we preferred spectral lines with no obvious blends and those strong enough to give reliable results even for spectra with lower $\mathrm{S} / \mathrm{N}$. Throughout this paper, we record the times of observations using reduced heliocentric Julian dates

RJD $=$ HJD -2400000.0.

The orbital solutions were obtained with the program FOTEL (Hadrava 2004a) and the combined orbital and light-curve solution for the eclipsing binary V1082 Sco was obtained with the latest version of program PHOEBE 1 (Prša \& Zwitter 2005, 2006). In a few cases, we also used the program KOREL for spectra disentangling (Hadrava 2004b). Having the Gaussian fits we could convert their widths to projected rotational velocities $v \sin i$. There are several studies that provide such conversion formulae for specific spectral lines. A suitable one for us is by Munari \& Tomasella (1999) for the He I $5876 \AA$ line. We prefer this particular line despite the fact that it is contaminated by several telluric lines since the blue parts of spectra are often noisier. This is particularly acute for the BESO spectra since the sensitivity (due to the optical fibre used at the time of our observations) dropped rapidly towards blue wavelengths.

\section{HD 92206 C}

The star CPD-57 $3580\left(\mathrm{CD}-57^{\circ} 3378\right.$, LS 1695) is often referred to as HD $92206 \mathrm{C}$, although it is not named so in WDS or SIMBAD. It is the second brightest member of the open cluster NGC 3324; the brightest member being HD 92206 AB. The open cluster is embedded in the $\mathrm{H}$ II region G 31. These two cluster members are separated by $36^{\prime \prime}$ and surrounded by groups of fainter stars. HD $92206 \mathrm{C}$ has $V=9^{\mathrm{m}} 05$ and a spectral type $\mathrm{O} 8 \mathrm{~V}+\mathrm{O} 9.7 \mathrm{~V}$ (according to Sota et al. 2014). The SB2 nature of this star was first reported by Campillay et al. (2007); they also found an orbital period of 2.02 days. No RVs had been published before.

There are ten BESO spectra of HD 92206 C; together with eight FEROS spectra from the ESO archive, they are listed in Table 2. RVs of both components were measured by Gaussian fitting in the He I $5876 \AA$ line, while only the primary is also visible in the He II $5411 \AA$ line. Therefore, the RVs measured for the latter line appear more representative for the primary RV curve. Narrow nebular lines are present in the Balmer as well as in the He I lines; due to the larger diameter of the BESO fiber, these emission lines are more pronounced there. Despite the low number of spectra, it was possible to find a period of 2.0225 . In the He I profiles, a third component is also clearly present, see Fig. 1; this component was not detected by Mason et al. (2009). As the diameter of the fiber in FEROS is $2^{\prime \prime}$, the component separation should be smaller than $1^{\prime \prime}$. There are indications that the RVs of this component are not constant. However, the superimposed nebular emission and a low $\mathrm{S} / \mathrm{N}$ of some spectra prevent us from finding the period of the third body.

Orbital elements of the close orbit are listed in Table 3 while the RV curve is shown in Fig. 2. In the epoch before reduced Julian date $(\mathrm{RJD}) \approx 55100$, the negative O-Cs prevail; after reduced Julian date (RJD), the positive ones are dominant. This might be a manifestation of the mutual orbit of the binary and the third body.

The equivalent widths (EWs) of the components in the He I $4922 \AA$ A line are 0.27 (primary), 0.21 (secondary) and $0.05 \mathrm{~A}$ (tertiary); the third component seems to be 2-3 mag fainter than the close binary suggesting a spectral type of B2. Assuming reasonable masses and radii, one could speculate that the objectmight be an ellipsoidal or even an eclipsing binary. The latter possibility is less likely, however, since no significant RossiterMcLaughlin effect was detected. Also, the observed $v \sin i$ seems to require a rather high orbital inclination in case of spin-orbital synchronisation, which is common among very close systems.

Recently, multicolour photometry of the object was obtained by Hackstein et al. (2015) and Dr. Hackstein kindly 


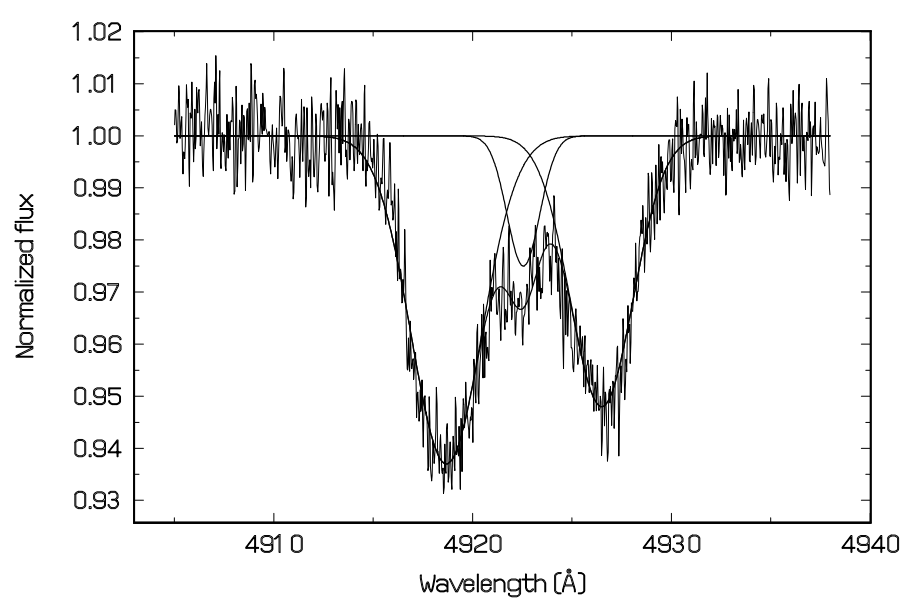

Fig. 1. Gaussian profiles of the primary, secondary and third components in the FEROS spectrum of HD 92206 C taken on RJD 54209.

Table 3. Orbital elements for HD 92206 C.

\begin{tabular}{lr}
\hline \hline Element & Value \\
\hline$P$ [days] & $2.022504(12)$ \\
$T_{\text {super.conj. }}$ [RJD] & $55901.9142(67)$ \\
$e$ & 0 (fixed) \\
$K_{1}\left[\mathrm{~km} \mathrm{~s}^{-1}\right]$ & $209.3(4.6)$ \\
$K_{2}\left[\mathrm{~km} \mathrm{~s}^{-1}\right]$ & $289.1(7.8)$ \\
$\gamma_{1}\left[\mathrm{~km} \mathrm{~s}^{-1}\right]$ & $-10.2(2.2)$ \\
$\gamma_{2}\left[\mathrm{~km} \mathrm{~s}^{-1}\right]$ & $1.2(7.8)$ \\
$m_{1} \sin ^{3} i\left[M_{\odot}\right]$ & $15.0(6)$ \\
$m_{2} \sin { }^{3} i\left[M_{\odot}\right]$ & $10.9(3)$ \\
$a \sin i\left[R_{\odot}\right]$ & $21.8(4)$ \\
$v_{1} \sin i\left[\mathrm{~km} \mathrm{~s}^{-1}\right]$ & $146(30)$ \\
$v_{2} \sin i\left[\mathrm{~km} \mathrm{~s}^{-1}\right]$ & $120(5)$ \\
$v_{3} \sin i\left[\mathrm{~km} \mathrm{~s}^{-1}\right]$ & $67(8)$ \\
$\mathrm{rms}_{1}\left[\mathrm{~km} \mathrm{~s}^{-1}\right]$ & 8.0 \\
$\mathrm{rms}_{2}\left[\mathrm{~km} \mathrm{~s}^{-1}\right]$ & 24 \\
\hline
\end{tabular}

Notes. All elements have their usual meaning; rms is the rms of one observation of unit weight derived from the difference between the calculated and observed RVs.

provided us with individual observations. Regrettably, only infrared magnitudes were not saturated. They have sufficient phase coverage, however, to exclude the presence of the binary eclipses. Further, they might indicate some low-amplitude physical variations, possibly associated with the rotational period of the primary, but their observational frequency was one observation per night. Therefore, new dedicated whole-night series of observations would be needed to (dis)prove the suspected physical light changes.

\section{HD 93146 A}

HD 93146 A (CPD-59 $2555, \mathrm{CD}-59^{\circ} 3278, \mathrm{LS} 1826 ; V=$ 8.44) is a member of the open cluster Collinder 228 (No. 65) in the Carina complex at a distance of approximately $2.3 \mathrm{kpc}$; the latter value depends on the adopted reddening law. Levato \& Malaroda (1981) determined a spectral type of O6 V and noted the variability of RVs; Walborn et al. (1985) suggested classification O6.5 V from IUE spectra. Recently, Sota et al. (2014; $R \sim 2500$ ) classified the star as O7 V((f))z. In our spectra the He II $4541 \AA$ line is clearly stronger than the He I $4471 \AA$ A line,

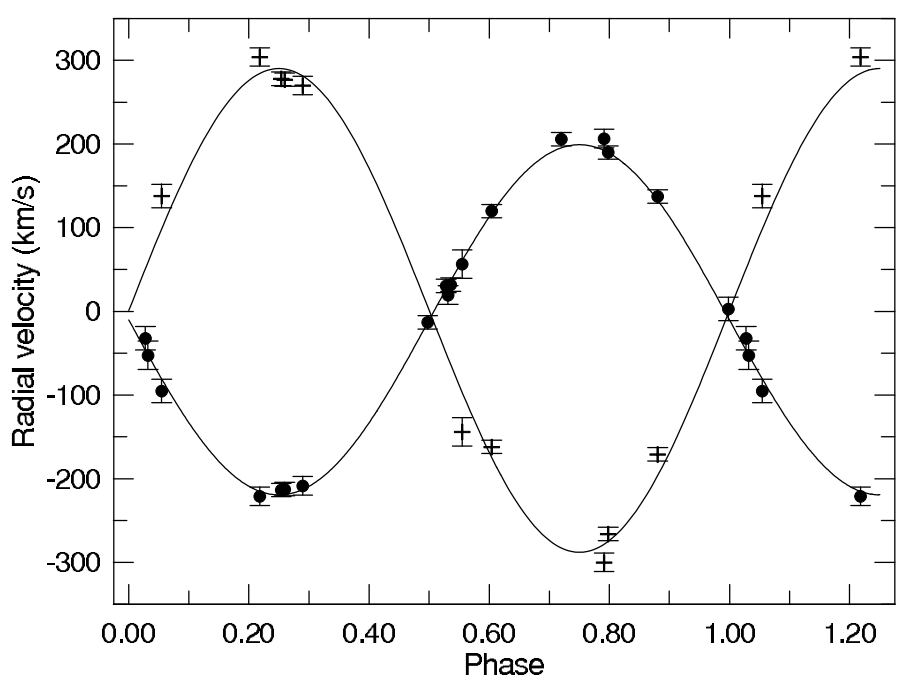

Fig. 2. Orbital RV curve of HD $92206 \mathrm{C}$ for the 2.0225 orbit.

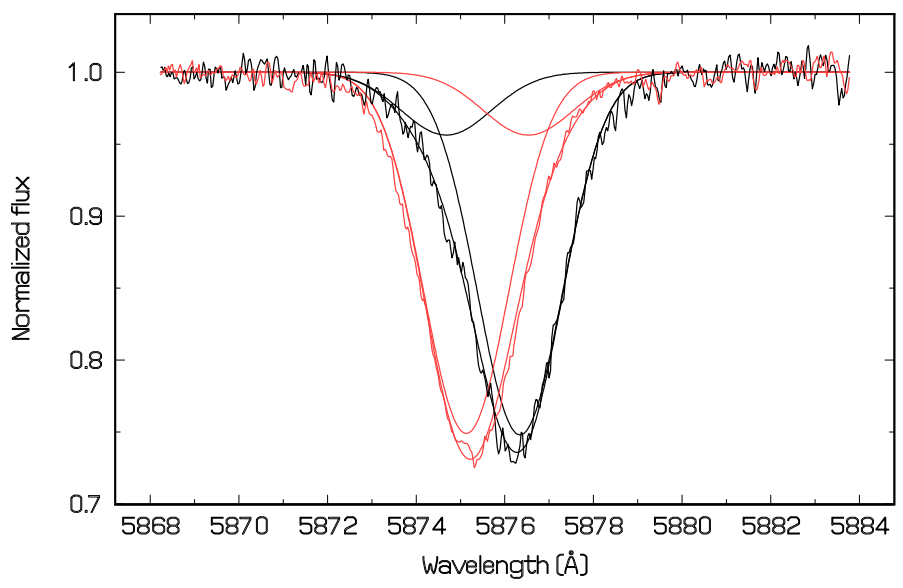

Fig. 3. Primary and secondary components of HD 93146 A in the line He I $5876 \AA ̊$ in spectra from RJD 54248 (black; phase 0.873) and 55643 (red; phase 0.111).

so the spectral type O6.5 V appears more appropriate. With this classification, the minimum mass of the primary (see Table 5) is also in better agreement with the expectation (Martins et al. 2005). However, we note that the He I lines are partly filled by the secondary component, which should be taken into account when classifying the spectral type. We also note that the Washington Double Star Catalogue (WDS Mason et al. 2001) lists a nearby (6".5) star that is 1.5 mag fainter than HD 93146 A. Sota et al. (2014) use the designation HD 93146 B and determine a spectral type of O9.7 IV. It is not clear at this point if the two stars form a physically bound system.

Levato et al. (1990) published ten RVs, some of which vary by $25 \mathrm{~km} \mathrm{~s}^{-1}$ from one day to another. In contrast, Sota et al. (2014) reported that OWN data suggest a period of 1130 days. We analysed seven BESO and twelve FEROS spectra and derived RVs using both the Gaussian fitting and asTODCOR; examples of the line profiles are in Fig. 3. Following the experience of Harmanec et al. (2015), we first disentangled the spectra using KOREL and made the preliminary orbital solution based on the RVs from the Gaussian fitting of line profiles. Then we used the disentangled spectra as templates for asTODCOR. A new version (R3) of asTODCOR was used, which also provides error estimates of individual RVs. According to the Appendix in Zucker et al. (1995), the error estimate of the component RVs 
P. Mayer et al.: Seven early-type binary and multiple systems

Table 4. RVs of HD 93146 A measured via Gaussian profiles and using asTODCOR over the spectral region from 4460 to $4723 \AA$.

\begin{tabular}{|c|c|c|c|c|c|c|}
\hline \multirow[t]{2}{*}{ RJD } & \multirow[t]{2}{*}{ Phase } & \multirow{2}{*}{$\begin{array}{l}5876 \\
\text { prim. }\end{array}$} & \multirow{2}{*}{$\begin{array}{l}5411 \\
\text { prim. }\end{array}$} & \multicolumn{2}{|c|}{ asTODCOR } & \multirow[t]{2}{*}{ Sp. } \\
\hline & & & & prim. & sec. & \\
\hline 53363.8162 & 0.0887 & -18.7 & -22.9 & $-28.62 \pm 0.18$ & $37.12 \pm 0.22$ & $\mathrm{~F}$ \\
\hline 54209.6500 & 0.8389 & 24.6 & 24.8 & $24.14 \pm 0.13$ & $-44.28 \pm 0.16$ & $\mathrm{~F}$ \\
\hline 54248.5217 & 0.8734 & 26.5 & 27.0 & $25.81 \pm 0.12$ & $-46.31 \pm 0.15$ & $\mathrm{~F}$ \\
\hline 54600.5969 & 0.1856 & -20.4 & -20.3 & $-22.98 \pm 0.10$ & $41.80 \pm 0.14$ & $\mathrm{~F}$ \\
\hline 54927.6812 & 0.4757 & -5.0 & -3.0 & - & - & B \\
\hline 55227.7110 & 0.7418 & 19.0 & 20.0 & $16.39 \pm 0.21$ & $-31.08 \pm 0.30$ & $\mathrm{~B}$ \\
\hline 55242.7476 & 0.7552 & 18.0 & 18.8 & $17.95 \pm 0.30$ & $-32.48 \pm 0.26$ & $\mathrm{~B}$ \\
\hline 55244.7298 & 0.7569 & 15.0 & 18.0 & $17.84 \pm 0.37$ & $-31.57 \pm 0.42$ & $\mathrm{~B}$ \\
\hline 55265.7237 & 0.7755 & 22.0 & 22.0 & $21.03 \pm 0.23$ & $-35.31 \pm 0.21$ & B \\
\hline 55291.5929 & 0.7985 & 26.0 & 27.0 & $21.77 \pm 0.23$ & $-39.16 \pm 0.18$ & B \\
\hline 55299.6685 & 0.8056 & 23.0 & 23.0 & $22.54 \pm 0.25$ & $-38.81 \pm 0.22$ & B \\
\hline 55323.5905 & 0.8269 & 25.9 & 26.3 & $24.80 \pm 0.12$ & $-41.71 \pm 0.14$ & $\mathrm{~F}$ \\
\hline 55325.5834 & 0.8286 & 23.6 & 24.0 & $24.26 \pm 0.10$ & $-43.29 \pm 0.12$ & $\mathrm{~F}$ \\
\hline 55326.5139 & 0.8295 & 25.3 & 25.7 & $24.49 \pm 0.10$ & $-43.12 \pm 0.12$ & $\mathrm{~F}$ \\
\hline 55604.8774 & 0.0763 & -18.0 & -17.9 & - & - & $\mathrm{F}$ \\
\hline 55641.6959 & 0.1090 & -22.9 & -23.7 & $-23.24 \pm 0.12$ & $41.69 \pm 0.18$ & $\mathrm{~F}$ \\
\hline 55643.6970 & 0.1108 & -26.5 & -27.0 & $-24.54 \pm 0.11$ & $41.74 \pm 0.13$ & $\mathrm{~F}$ \\
\hline 55698.6435 & 0.1595 & -23.6 & -23.4 & $-24.28 \pm 0.13$ & $42.72 \pm 0.17$ & $\mathrm{~F}$ \\
\hline 56068.5713 & 0.4876 & -1.9 & -1.9 & $-3.02 \pm 0.11$ & $4.82 \pm 0.15$ & $\mathrm{~F}$ \\
\hline
\end{tabular}

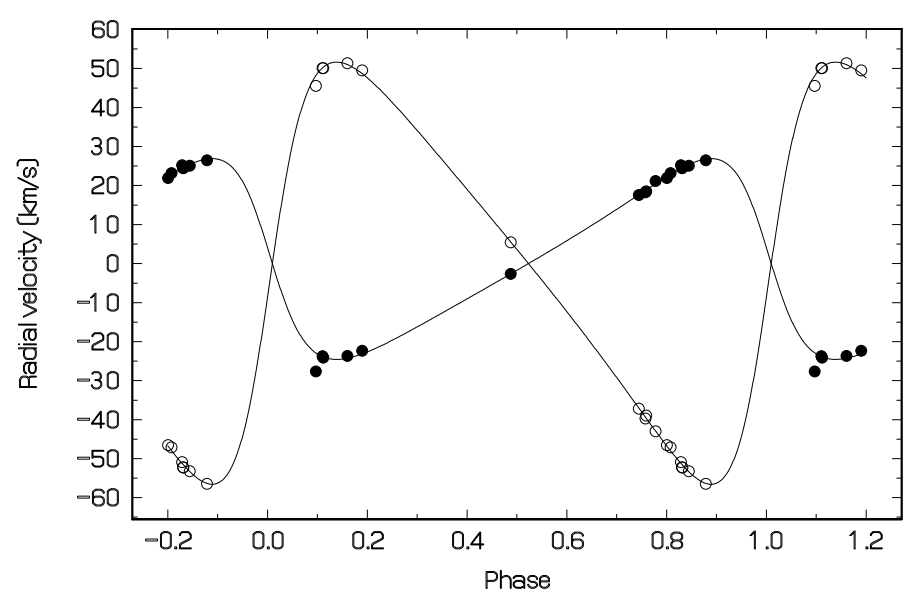

Fig. 4. Orbital RV curves of HD 93146 based on asTODCOR RVs. The estimated errors are generally comparable to the size of the symbols (black dots and open circles for the primary and secondary, respectively), and are not, therefore, shown.

derived from multi-dimension cross-correlation techniques can be obtained from a one dimensional (1D) error analysis carried out on each dimension. In this version of the program, we therefore applied a relation deduced by Zucker (2003) from the maximum-likelihood theory that provides reliable estimates of the uncertainty as long as the errors are dominated by random processes.

With both methods we could derive RVs also for the secondary component from the He I $5876 \AA$ line by fitting the Gaussians, and in the spectral region from 4460 to $4723 \AA$ using asTODCOR. The spectra and RVs measured by both techniques are listed in Table 4. For the orbital solution, we used the asTODCOR RVs individually weighted by weights inversely proportional to the square of their estimated errors. The orbital RV curve is shown in Fig. 4. The orbital solution is in Table 5 and corroborates a long period of 1131.6 days.
Table 5. Orbital elements for HD 93146 A; same notation as in Table 3.

\begin{tabular}{lr}
\hline \hline Element & Value \\
\hline$P$ [days $]$ & $1131.4(2.9)$ \\
$T_{\text {periastr. }}[\mathrm{RJD}]$ & $54385.9(6.6)$ \\
$e$ & $0.4069(87)$ \\
$\omega\left[^{\circ}\right]$ & $83.6(1.7)$ \\
$K_{1}\left[\mathrm{~km} \mathrm{~s}^{-1}\right]$ & $25.73(37)$ \\
$K_{2}\left[\mathrm{~km} \mathrm{~s}^{-1}\right]$ & $54.10(1.05)$ \\
$\gamma_{1}\left[\mathrm{~km} \mathrm{~s}^{-1}\right]$ & $-0.41(0.41)$ \\
$\gamma_{2}\left[\mathrm{~km} \mathrm{~s}^{-1}\right]$ & $+0.35(0.70)$ \\
$m_{1} \sin ^{3} i\left[M_{\odot}\right]$ & $30.81(96)$ \\
$m_{2} \sin ^{3} i\left[M_{\odot}\right]$ & $14.66(48)$ \\
$a \sin i\left[R_{\odot}\right]$ & $1631(31)$ \\
$v_{1} \sin i\left[\mathrm{~km} \mathrm{~s}^{-1}\right]$ & $62(4)$ \\
$v_{2} \sin i\left[\mathrm{~km} \mathrm{~s}^{-1}\right]$ & $50(10)$ \\
$\mathrm{rms}_{1}\left[\mathrm{~km} \mathrm{~s}^{-1}\right]$ & 0.91 \\
$\mathrm{rms}_{2}\left[\mathrm{~km} \mathrm{~s}^{-1}\right]$ & 0.61 \\
\hline
\end{tabular}

Notes. The spectra disentangled by KOREL have RVs corresponding to the systemic velocity. When used as templates for asTODCOR, RVs corresponding to zero systemic velocity are obtained.

The EWs of both components for the He I $5876 \AA$ line are 0.55 and $0.09 \AA$, respectively, which implies a spectral class of approximately B0.5 for the secondary. If the visible O9.7 IV neighbour (HD 93146 B) were indeed physically bound, HD 93146 A would be another hierarchical triple system of highmass stars.

\section{HD 112244}

HD 112244 (HD 112244A, HR 4908, CD-56²4699, HIP 63117 and $54 \mathrm{G} \mathrm{Cru}$ ) has the spectral type O8.5 Iab(f)p (Sota et al. 2014) and a visual brightness of $V=5 \mathrm{~m} .38$. It has been part of the monitoring program for stellar multiplicity (Chini et al. 2012) and attracted our attention because of its highly variable spectral line profiles. 

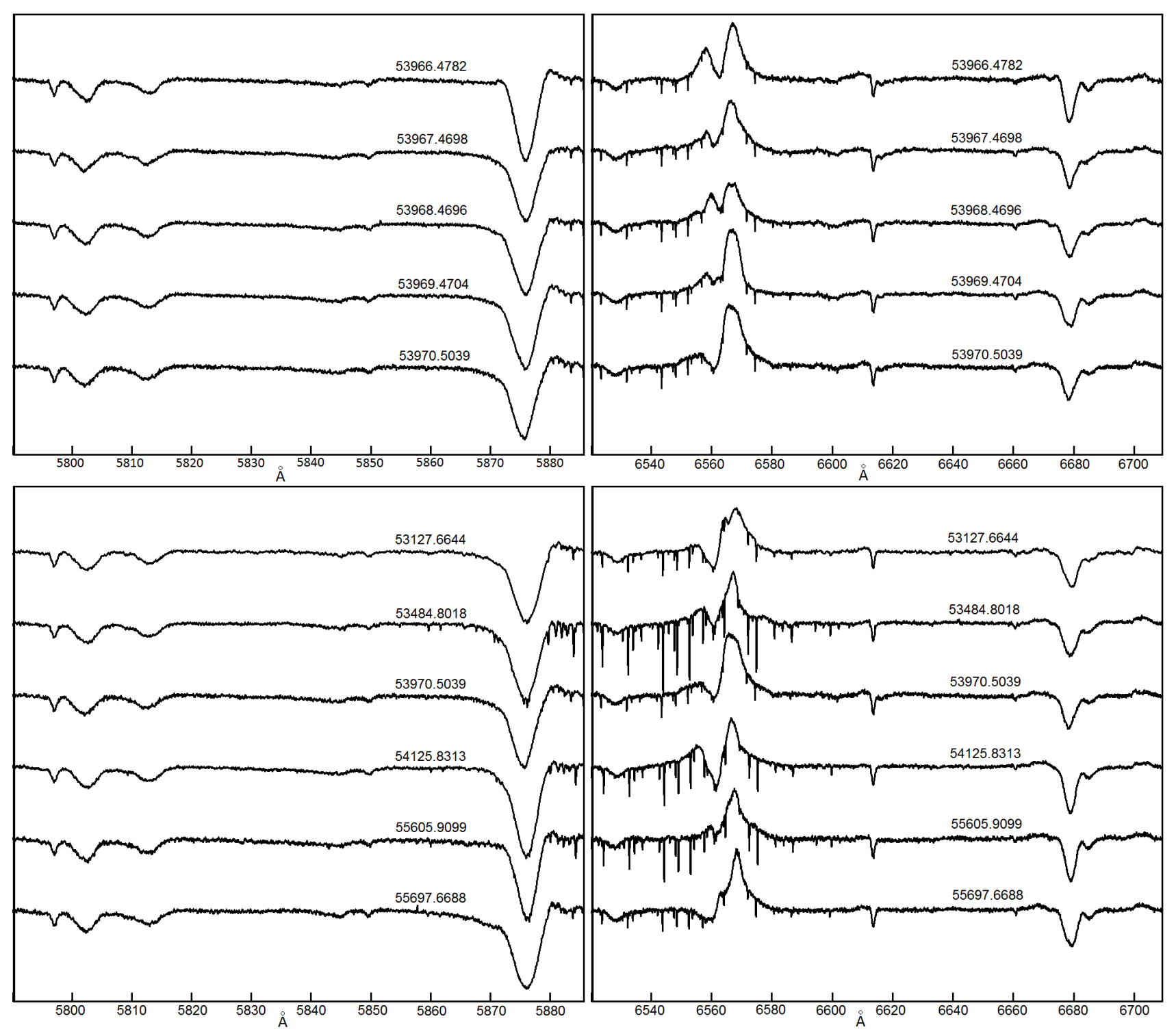

Fig. 5. Examples of variability of line profiles originating in the circumstellar matter in the yellow and red parts of the spectra of HD 112244. Left panels show the diffuse interstellar band at $5797.0 \AA$, the C IV 5801.3 and $5812.0 \AA$, and the He I $5875.7 \AA$ line profiles, while the right panels show $\mathrm{H} \alpha$, the diffuse interstellar $6613.6 \AA$ band and the He I $6678.2 \AA$ and He II $6683 \AA$ line profiles for the same spectra. The upper panels illustrate the variability on a time scale of days and the bottom panels show variations on longer time scales. The offset of individual profiles is 0.25 of the continuum level.

Radial velocities were measured by several investigators but no periodicity of RV changes was reported. Stickland \& Lloyd (2001) measured RVs in 9 IUE spectra and tried to search for periodicity in them and in previously published RVs, but could not find any. They concluded that the observed RV changes are most likely due to atmospheric instabilities. Sota et al. (2014) in their GOSSS survey mention that according to a parallel high-dispersion OWN survey (Barbá et al. 2010), HD 112244 is a double-lined spectroscopic binary with a $7 \mathrm{~d} 5$ period. They remarked that they do not see double lines in the GOSSS spectra.

Jakate (1979), in his search for possible new $\beta$ Cep variables, found HD 112244 constant on six nights of his observations. However, the range of variations found by the HIPPARCos satellite $H_{\mathrm{p}}$ photometry from 5.33 to $5 \cdot 41$ (Perryman \& ESA 1997) seems to indicate some micro-variability. Indeed, Marchenko et al. (1998) analysed $H_{\mathrm{p}}$ photometry and suspected the presence of three possible periods, $4 \mathrm{~d} 518,3.286$ or $1 \mathrm{~d} 156$, the amplitude of variations being slightly over 0.02 in all three cases. Repeated analysis of the same data by Koen \& Eyer (2002) resulted in finding a period of 2.00288 with a semiamplitude of 0.0085 .

Our observational material consists of 68 BESO and 30 FEROS spectra secured between May 2004 and August 2014 (RJD 53 127-56880). Ten FEROS spectra secured between RJD 53898.6647 and 53919.5607 have exposure times of $9 \mathrm{~s}$ only and are relatively noisy. Also, the last series of BESO spectra starting with RJD 56854.4909 was obtained in very adverse weather conditions and has a lower quality. For that reason, we measured the $\mathrm{S} / \mathrm{N}$ for each spectrum and applied weights proportional to the square of $\mathrm{S} / \mathrm{N}$ and normalised to the mean $\mathrm{S} / \mathrm{N}$.

HD 112244 is an emission-line object and has strong stellar wind. Martins et al. (2015) have shown that rapid and apparently irregular variability of the wind line profiles is characteristic of the OB supergiants. This is also true for HD 112244 as illustrated in Fig. 5, where we show parts of the yellow and red spectra for 


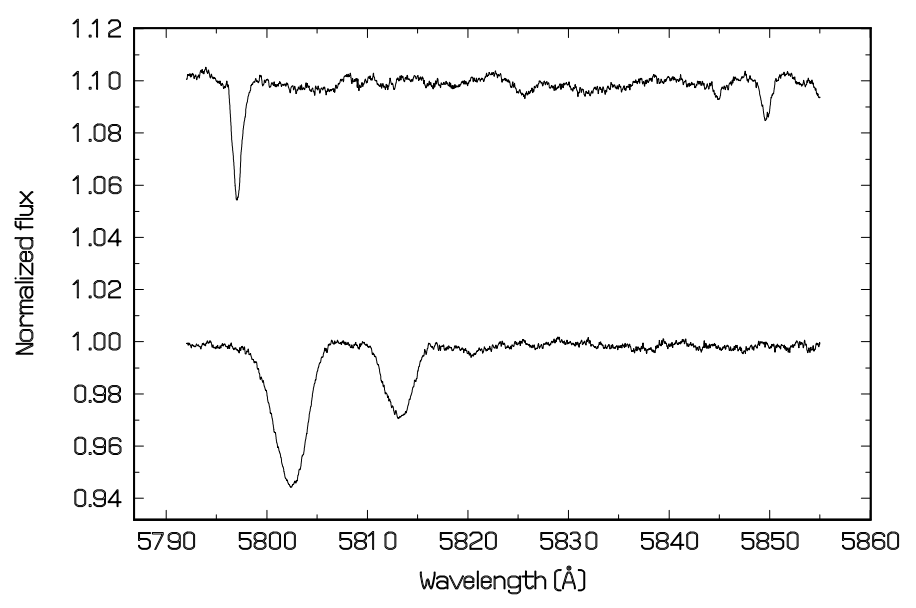

Fig. 6. Disentangled spectrum of HD 112244 and of diffuse interstellar bands in the wavelength region 5792-5855 A.

selected spectrograms. It is seen that the strength and shape of the $\mathrm{H} \alpha$ line and also of the faint emission at the red wing of the He I $5876 \AA$ line both vary on timescales from days to years, while the line profiles of high-ionisation species as He II or C IV remain relatively stable. This fact represents a complication for RV measurements since even seemingly photospheric lines can be partly affected by these physical variations.

To check whether HD 112244 is indeed a spectroscopic binary, we measured RVs of several spectral lines using different techniques. The RVs of basically photospheric lines of

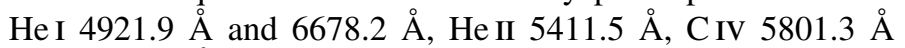
and $5812.0 \AA$, and of two lines affected by emission $(\mathrm{H} \alpha$ and He I $5875.7 \AA$ ) were measured via a comparison of direct and flipped line profiles in SPEFO. The RV of the clean He II $5411.5 \AA$ line was also measured through a Gaussian fit of the profile. Using Stellingwerf (1978) phase dispersion minimisation period search technique, we analysed all these RV data subsets for periodicity over the frequency range down to $10 \mathrm{~cd}^{-1}$. In all cases, this led to the detection of a dominant period of 27.67 and RV curves indicative of a highly eccentric orbit.

For the final analysis, we selected the region from 5792 to $5855 \AA$ and proceeded in the following way. We formally disentangled the stellar spectrum of the C IV 5801 and $5812 \AA$ and the spectrum of diffuse interstellar lines. These disentangled spectra, shown in Fig. 6, were then used as templates for the 2D crosscorrelation with the program asTODCOR.

Figure 7 shows the phase-dispersion periodogram for asTODCOR RVs down to a period of 0.2 . One can see that the period of 27.67 (frequency $0.036 \mathrm{c} \mathrm{d}^{-1}$ ) represents the dominant minimum in the periodogram. Other deeper minima can be identified with the second harmonic at $0.072 \mathrm{c} \mathrm{d}^{-1}, 1 \mathrm{~d}$ alias at $0.963 \mathrm{c} \mathrm{d}^{-1}$ and $1 \mathrm{yr}$ alias at $0.039 \mathrm{~cd}^{-1}$. We note that there is no evidence of the earlier reported 7.5 period $\left(f=0.133 \mathrm{c} \mathrm{d}^{-1}\right)$. Our orbital solution based on these asTODCOR RVs with weights proportional to the square of the $\mathrm{S} / \mathrm{N}$ is summarized in Table 6 .

The secondary must be much less massive and indeed our attempts to disentangle it failed. The corresponding RV curve is depicted in Fig. 8. It is clear that larger deviations from the curve come invariably from spectra of lower quality. Regrettably, no RVs of superb quality are available at the bottom part of the RV curve. We postpone the discussion of binary properties to the future, when a dedicated series of well exposed spectra will be obtained. Nevertheless, a crude estimate of the secondary mass
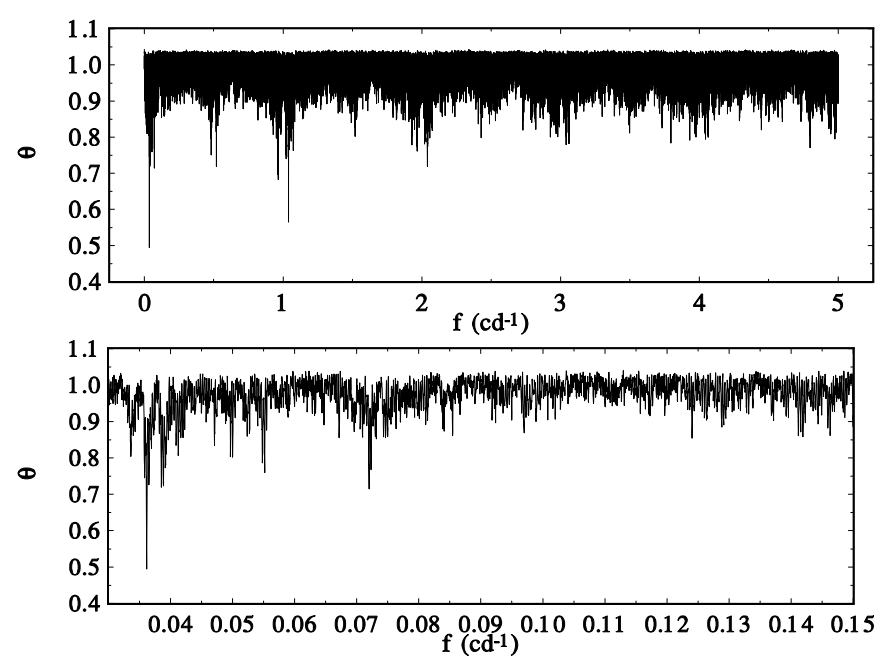

Fig. 7. Stellingwerf's $\theta$ statistics periodogram for asTODCOR RVs from the 5792-5855 A region. A complete periodogram for periods down to 0.2 is shown in the upper panel and a zoomed part of it in the bottom one.

Table 6. Orbital elements for HD 112244; same notation as in Table 3.

\begin{tabular}{lr}
\hline \hline Element & Value \\
\hline$P$ [days] & $27.6652(22)$ \\
$T_{\text {periastr. }}[\mathrm{RJD}]$ & $55532.20(21)$ \\
$e$ & $0.692(50)$ \\
$\omega\left[{ }^{\circ}\right]$ & $290.8(7.5)$ \\
$K_{1}\left[\mathrm{~km} \mathrm{~s}^{-1}\right]$ & $17.4(1.9)$ \\
$f(m)\left[M_{\odot}\right]$ & $0.00572(22)$ \\
$v_{1} \sin i\left[\mathrm{~km} \mathrm{~s}^{-1}\right]$ & $122-166^{1}$ \\
$\mathrm{rms}\left[\mathrm{km} \mathrm{s}^{-1}\right]$ & 5.8 \\
\hline
\end{tabular}

Notes. (1) The interval corresponds to full width at half maximum (FWHM).

can be obtained from the mass function $f(m)$. Adopting $m_{1}=$ 31.54 for O8.5 I (Martins et al. 2005), we obtain a mass range of $1.85-3.85 M_{\odot}$ for a range of inclinations from $90^{\circ}$ to $30^{\circ}$.

The periodic RV variability of the stellar lines seems to be beyond doubt, especially if compared to the RVs of the diffuse interstellar bands. The light variations plotted vs. orbital phase appear to be indicative of some co-rotating structures, also known for some other OB stars (cf., e.g. Owocki et al. 1995; Harmanec 1989, 1999). More observations are required, however, to prove or disprove this classification.

\section{HD 123056}

HD $123056\left(\mathrm{CPD}-59^{\circ} 5404, \mathrm{CD}-59^{\circ} 5090, \mathrm{LS} 3200\right)$ is a field star with $V=8.14$ and was classified as $09.5 \mathrm{~V}((\mathrm{n}))$ (Walborn 1973); Sota et al. (2014) give an integral spectral type of O9.5 IV(n). Feast et al. (1963) published four RVs and noted their variability; Chini et al. (2012) found varying double lines and assigned the status SB2. Sota et al. (2014) suggested that the star is "at least SB2", speculating that it might also be a triple or multiple system.

We could secure ten spectra with BESO; five more spectra were obtained within the program "Tycho Brahe" in May 2015, when the $2.2 \mathrm{~m}$ MPI telescope was allocated to programs proposed by Czech astronomers (see Table 7). Furthermore, there are eight FEROS spectra in the ESO archive. The profiles of the 

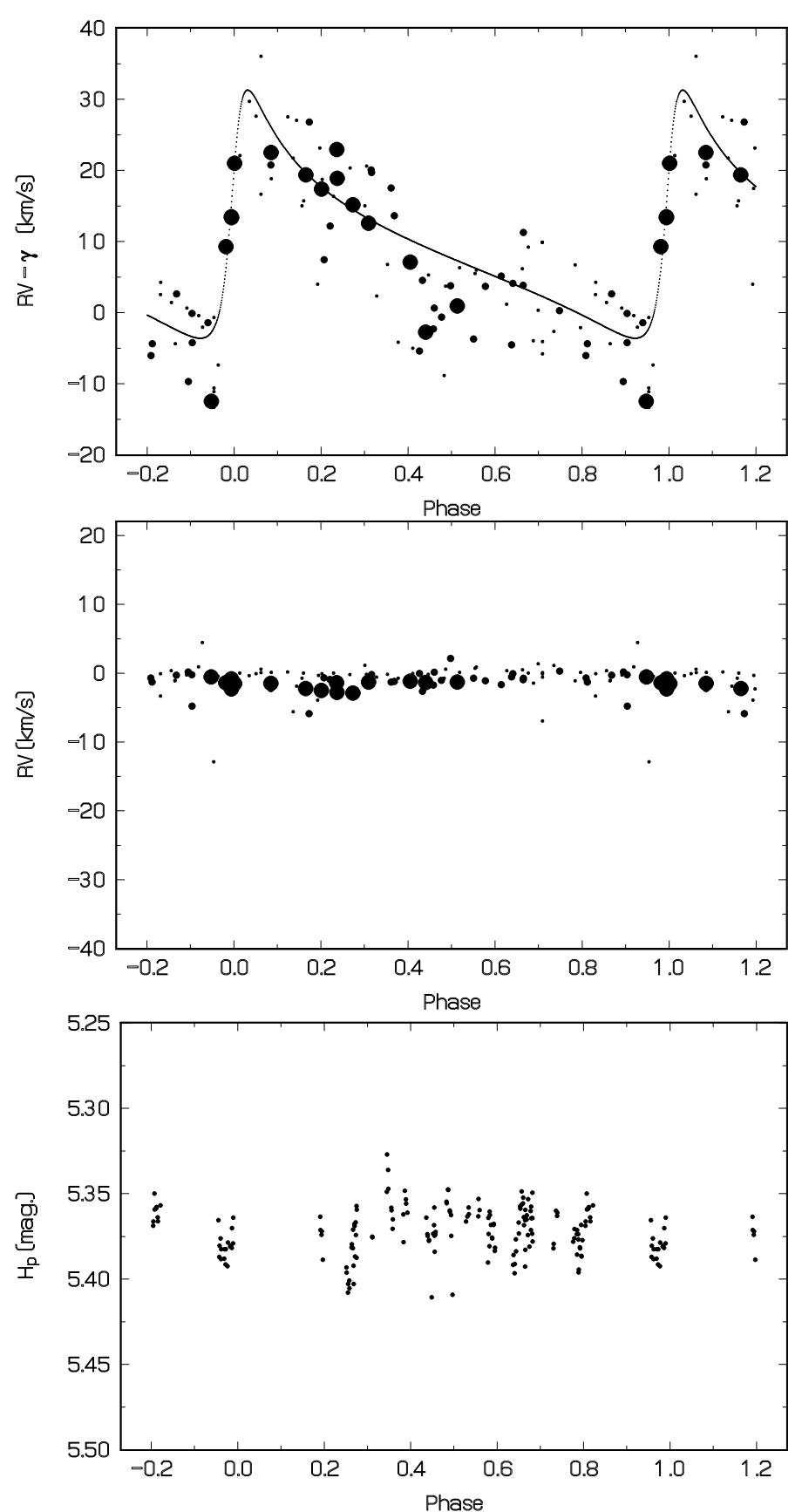

Fig. 8. Upper panel: orbital RV curve plot of HD 112244 based on asTODCOR RVs from the 5792-8555 $\AA$ region. RVs from spectra with weights higher than 1.5 are shown by large black circles, those with weights between 1 and 1.5 with smaller black circles, and those with weights lower than 1 with black dots. Middle panel: asTODCOR RVs of the diffuse absorption bands shown on the same scale to characterise the uncertainties of the RV determination. The same symbols as above. Bottom panel: HIPPARCos $H_{\mathrm{p}}$ magnitude plotted vs. orbital phase.

He I $6678 \AA$ and the He II $5411 \AA$ lines (FEROS spectra) are shown in Fig. 9.

As the He II $5411 \AA$ line is broad and any indications for duplicity are virtually missing, we conclude that it belongs to a third component, hereafter referred to as " $\mathrm{A}$ ". From its position changes, we conclude that A has a variable RV. The number of spectra is not sufficient for a reliable determination of the period. With the Radcliffe observatory spectra included, the period-finding program found a period near 1310 days as the best one and the free orbital solution improved this value to $1314.1 \pm 1.8 \mathrm{~d}$; the probable orbital elements are listed in Table 8 . We admit that this must not be the only possible solution, since some of the RVs are not completely reliable. Components of the binary B are distinguishable near elongations of their orbit in the He I lines (cf. Fig. 9).

It is not clear whether this long period is that of a binary or of the third body orbit (A) around the closer binary (B). Nevertheless, the body with the long period must be of earlier spectral type (say $\approx 08.5$ ). As the components of the binary $\mathrm{B}$ are better seen in the He I lines (are invisible in He II $5411 \AA$ ), they are of type $\approx \mathrm{B} 0$; a discrimination between components $\mathrm{B} 1$ and $\mathrm{B} 2$ is difficult. The amplitudes of RVs in all orbits are rather small, therefore the accuracy of the derived periods is also affected. The period of the binary B seems to be slightly shorter than 2 days. To summarise, HD 123056 appears to be another hierarchical triple system of high-mass stars.

\section{HD 164438}

HD 164438 (BD-194800, LS 4567) is a star in the Sgr OB1 association with $V=7.48$ and a spectral type of O9.2 IV (Sota et al. 2014). Mason et al. (1998) found a possible binary companion at a separation of $00^{\prime \prime} 05$, which, however, needs confirmation as it was not seen by Sana et al. (2014). Chini et al. (2012) classified HD 164438 as SB1, which was recently corroborated by OWN data (Sota et al. 2014).

We were able to secure 15 BESO spectra and downloaded 5 FEROS spectra from the ESO archive. The RVs were measured from the He I $5876 \AA$ and He II $5411 \AA$ lines via Gaussian fits. The corresponding results are listed in Table 9, the orbital elements are given in Table 10, while the RV curve is shown in Fig. 11. When inspecting the line profiles, one cannot find any trace of the lines from the secondary. We attempted to detect the secondary through disentangling with KOREL, but without success. This means that the EWs of the secondary component must be approximately 20 times smaller than those of the primary, implying a spectral type later than B3. Using the mass function $f(m)$ and adopting $m_{1}=19 M_{\odot}$ for O9.2 IV (as can be obtained by intepolating in tables by, e.g. Martins et al. 2005) we obtain a mass range of 2.02-4.32 $M_{\odot}$ for a range of inclinations from $90^{\circ}-30^{\circ}$.

\section{HD 164816}

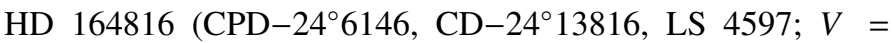
7.08 ) is located towards the young open cluster NGC 6530. It was originally classified as O9.5 III-IV(n) (Walborn 1973). The spectral type B3 Ve assigned by Levenhagen \& Leister (2006) must be a mistake, since the O-type character of the spectrum is without any doubt; the emission is present as narrow lines, so it originates from the surrounding $\mathrm{H}$ II region. Chini et al. (2012) classified HD 164438 as SB2. Double lines are also seen by Sota et al. (2014), who derived spectral types of O9.5 V + B0 V for the two components.

Trepl et al. (2012) have published an extensive multiwavelength (X-ray, $\gamma$-ray, optical and radio) study of the binary HD 164816. They find an orbital period of 3.82 days and projected masses of $m_{1} \sin ^{3} i=2.355(69) M_{\odot}$ and $m_{2} \sin ^{3} i=$ 2.103(62) $M_{\odot}$. They also identified an associated X-ray source with a soft X-ray excess and a $10 \mathrm{~s}$ pulsation, which they interpret as the signature of a neutron star in the system. 
P. Mayer et al.: Seven early-type binary and multiple systems

Table 7. SPEFO RVs of the wings and two line cores of the He I $5876 \AA$ line and RVs from Gaussian fits of the He II $5411 \AA$ A line of HD 123056 (in $\mathrm{km} \mathrm{s}^{-1}$ ).

\begin{tabular}{crrrrrrc}
\hline \hline RJD & $\begin{array}{r}5876 \\
\text { wings }\end{array}$ & $\begin{array}{r}\text { O-C } \\
\text { core }\end{array}$ & $\begin{array}{r}5876 \\
\text { 2nd core }\end{array}$ & 5876 & 5411 & O-C & $\begin{array}{c}\text { Spectro- } \\
\text { graph }\end{array}$ \\
\hline 34825.5256 & -30.00 & -5.0 & & & & & $\mathrm{R}$ \\
34834.4991 & -23.00 & 3.3 & & & & & $\mathrm{R}$ \\
35181.5451 & -59.00 & -8.3 & & & & & $\mathrm{R}$ \\
35208.4685 & -44.00 & 5.0 & & & & & $\mathrm{R}$ \\
54935.6907 & -38.23 & 9.7 & -2.10 & - & -36 & 9.8 & $\mathrm{~B}$ \\
54952.7769 & -53.41 & -6.8 & -3.59 & - & -48 & -3.3 & $\mathrm{~B}$ \\
54954.7400 & -52.74 & -6.2 & 19.43 & -88.48 & -53 & -8.4 & $\mathrm{~F}$ \\
54960.7492 & -48.83 & -2.8 & -47.60 & - & -51 & -6.8 & $\mathrm{~B}$ \\
54976.6593 & -39.31 & 5.5 & -49.72 & - & -38 & 5.1 & $\mathrm{~F}$ \\
55240.9051 & -10.76 & 12.1 & 28.73 & -68.46 & -12 & 10.9 & $\mathrm{~B}$ \\
55241.9183 & -18.94 & 3.8 & 14.42 & - & -23 & -0.2 & $\mathrm{~B}$ \\
55251.8066 & -19.02 & 3.0 & -31.34 & - & -22 & 0.1 & $\mathrm{~B}$ \\
55253.8783 & -35.55 & -13.7 & 19.24 & -96.36 & -28 & -6.1 & $\mathrm{~B}$ \\
55605.8938 & -11.34 & -4.6 & 7.84 & -89.40 & -13 & -7.8 & $\mathrm{~F}$ \\
55642.7878 & -14.63 & -7.3 & -3.83 & - & -11 & -5.2 & $\mathrm{~F}$ \\
55697.7150 & -1.42 & 8.0 & -60.12 & 22.53 & -2 & 6.2 & $\mathrm{~F}$ \\
55698.7538 & -5.48 & 4.0 & -4.33 & - & -5 & 3.2 & $\mathrm{~F}$ \\
55794.9821 & -16.54 & 1.0 & 3.44 & - & -15 & 2.8 & $\mathrm{~B}$ \\
55803.9761 & -13.59 & 5.0 & -37.69 & - & -16 & 3.1 & $\mathrm{~B}$ \\
55805.9746 & -12.87 & 6.0 & 7.33 & - & -7 & 12.3 & $\mathrm{~B}$ \\
56067.7858 & -48.14 & 4.2 & -57.94 & - & -45 & 5.5 & $\mathrm{~F}$ \\
56068.7840 & -52.54 & -0.1 & -4.48 & - & -53 & -2.5 & $\mathrm{~F}$ \\
57155.8197 & -24.22 & -0.6 & -41.14 & 29.73 & -33 & -8.3 & $\mathrm{~F}$ \\
57156.8305 & -27.45 & -3.7 & -38.31 & - & -28 & -3.2 & $\mathrm{~F}$ \\
57157.7282 & -19.56 & 4.4 & 24.60 & -88.29 & -27 & -2.0 & $\mathrm{~F}$ \\
57159.7152 & -26.52 & -2.3 & 18.48 & - & -26 & -0.7 & $\mathrm{~F}$ \\
57160.5437 & -33.10 & -8.8 & -56.91 & - & -30 & -4.6 & $\mathrm{~F}$ \\
\hline
\end{tabular}

Notes. The four early Radcliffe RVs (R) are the mean RVs of several spectral lines. To estimate the measurement errors of the components of He I $5876 \AA$ in SPEFO, we repeated the measurements several times independently. The results for the wings usually differed by less than $1 \mathrm{~km} \mathrm{~s}^{-1}$, while the settings for the line cores are less certain, especially for lower S/N BESO spectra.

During an interferometric infrared survey, Sana et al. (2014) detected a faint companion separated by 57 mas. This object is not a component of the SB2 system itself because this pair is separated by only 0.07 mas assuming a distance of $1 \mathrm{kpc}$ as suggested by Trepl et al. (2012). The difference of the $H$ magnitude of HD 164816 and the faint companion is $\Delta H=3.4$, suggesting quite different masses. Because the object is also detected in X-rays, Sana et al. (2014) suggested that they probably see an active later-type object, which may provide an alternative explanation to the X-ray excess of HD 164816.

We re-analysed the existing BESO spectra and also included two FEROS spectra from the ESO archive in our analysis. In general, we can confirm the orbital elements published by Trepl et al. (2012). However, we obtain a systemic velocity equal to the velocity of the cluster. We suspect that in the cited paper the spectra were not reduced correctly. Note that Stickland \& Lloyd (2001) published the following RVs (in $\mathrm{km} \mathrm{s}^{-1}$ )

RJD $43782.337: V_{1}=39.7, V_{2}=-34.5$,

RJD $44899.205: V_{1}=77.7, V_{2}=-81.9$,

which clearly suggest that the systemic velocity is close to zero (or close to the RV of the cluster, which is approximately $-7 \mathrm{~km} \mathrm{~s}^{-1}$ ). We believe that the reason why Trepl et al. (2012) obtained an anomalous systemic velocity lies in the fact that they overlooked that the first pixel of BESO spectra is numbered -51 in their reduction and therefore the spectra must be shifted by $1.56 \AA$. For the He I $5876 \AA$ line, this corresponds to $78 \mathrm{~km} \mathrm{~s}^{-1}$, which is close to the value of the erroneous systemic velocity published by Trepl et al. (2012).

As a consequence, the distance of the binary might well be equal to the distance of NGC 6530 whose latest determinations are $1250 \mathrm{pc}$ (Prisinzano et al. 2005) and $1322 \mathrm{pc}$ (Kharchenko et al. 2005), respectively. Likewise, the cluster was found to be affected by anomalous extinction with $R=4.5$ (Fernandes et al. 2012). Although SIMBAD classifies HD 164816 as a Be star (Levenhagen \& Leister 2006), it seems that among eight sources of $U B V$ photometry there are no pronounced light or colour changes observed. We therefore used mean photometric values $V=7.092$ and $B-V=0.02$ (Mermilliod 1986). De-reddening leads to $E_{B-V}=0.292,(B-$ $V)_{0}=-0.29$ and $(U-B)_{0}=-1.09$ resulting in colours that agree well with an $\mathrm{O} 9.5 \mathrm{~V}+\mathrm{B} 0 \mathrm{~V}$ spectrum. At a distance of $14 \mathrm{arcmin}$ from the cluster centre and a cluster radius of $15 \mathrm{arcmin}$, the star is located at the outskirts of NGC 6530. Using $R=4.5$ to derive the visual extinction yields $V_{0}=5.78$, while a normal value of $R=3.1$ would imply $V_{0}=6.19$.

To derive the absolute visual magnitude of the system, we calculated the sum $M_{V}^{\mathrm{pri}}+M_{V}^{\mathrm{sec}}=-4.58$ with $M_{V}^{\mathrm{pri}}=-3.90$ for O9.5 $\mathrm{V}$ and $M_{V}^{\mathrm{sec}}=-3.75$ for B0 V; the latter value was extrapolated from Martins et al. (2005). Eventually this leads to a distance modulus of 10.36 or 10.77 for $R=4.5$ or 3.1 , respectively. 


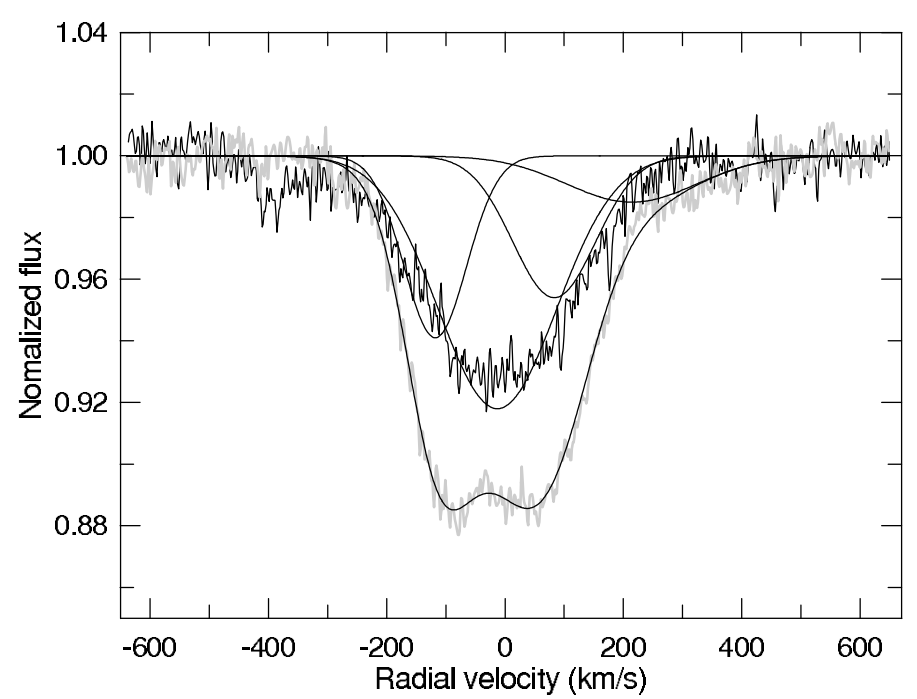

Fig. 9. An attempt to resolve the profile of the line He I $6678 \AA$ in HD 123056 obtained at RJD 54960 . The RV of the central line of the profile (binary A) has been assumed to be identical to the RV measured for the line He II $5411 \AA$. The thick line shows the He II line 5411 (with DIBs at approximately +390 and $-390 \mathrm{~km} \mathrm{~s}^{-1}$ ). The grey line is the observed line He I $6678 \AA$ and the thin lines represent components of the binary B. The line He II $6683 \AA$ in the binary A is also present.

Table 8. Probable orbital elements of the long AB orbit of HD 123056; same notation as in Table 3.

\begin{tabular}{lr}
\hline \hline Element & Value \\
\hline$P$ [days] & $1314.1(1.8)$ \\
$T_{\text {periastr. }}[\mathrm{RJD}]$ & $54648(95)$ \\
$e$ & $0.182(70)$ \\
$K_{1}\left[\mathrm{~km} \mathrm{~s}^{-1}\right]$ & $23.4(2.7)$ \\
$\gamma_{1}\left[\mathrm{~km} \mathrm{~s}^{-1}\right]$ & $-28.0(1.5)$ \\
$f(m)\left[M_{\odot}\right]$ & $1.66(72)$ \\
$a_{1} \sin i\left[R_{\odot}\right]$ & $598(76)$ \\
$v_{1} \sin i\left[\mathrm{~km} \mathrm{~s}^{-1}\right]$ & $140(20)$ \\
$v_{2} \sin i\left[\mathrm{~km} \mathrm{~s}^{-1}\right]$ & $140(20)$ \\
$v_{3} \sin i\left[\mathrm{~km} \mathrm{~s}^{-1}\right]$ & $225(30)$ \\
$\mathrm{rms}_{1}\left[\mathrm{~km} \mathrm{~s}^{-1}\right]$ & 5.85 \\
\hline \multicolumn{2}{c}{} \\
\hline
\end{tabular}

The corresponding distances of $1180 \mathrm{pc}$ and $1426 \mathrm{pc}$ display the influence of the extinction law but nicely embrace the latest determinations for NGC 6530. Our estimate is also supported by the latest Gaia measurements (Gaia Collaboration 2016) $d=$ $1100 \pm 530 \mathrm{pc}$. Therefore, HD 164816 might well be a cluster member.

The rate of the apsidal advance is given as 0.67(7) $\mathrm{rad} \mathrm{yr}^{-1}$ by Trepl et al. (2012). A binary that has a similar spectral type and orbital period is Y Cyg. Its apsidal motion is much slower, $0.1314 \mathrm{rad} \mathrm{yr}^{-1}$ (see, e.g. Harmanec et al. 2014, and references therein); there are no obvious reasons for such a huge difference between those two binaries. Indeed, although our spectra now cover a longer time interval than those used by Trepl et al. (2012), we were unable to find any evidence for apsidal motion.

\section{HD 318015 (V1082 Sco)}

This star belongs to the rare class of eclipsing binaries containing a supergiant. It was classified as B0.7 Ia by Massey et al. (2001), B0.5 Ib by Moffat et al. (1977) and also independently

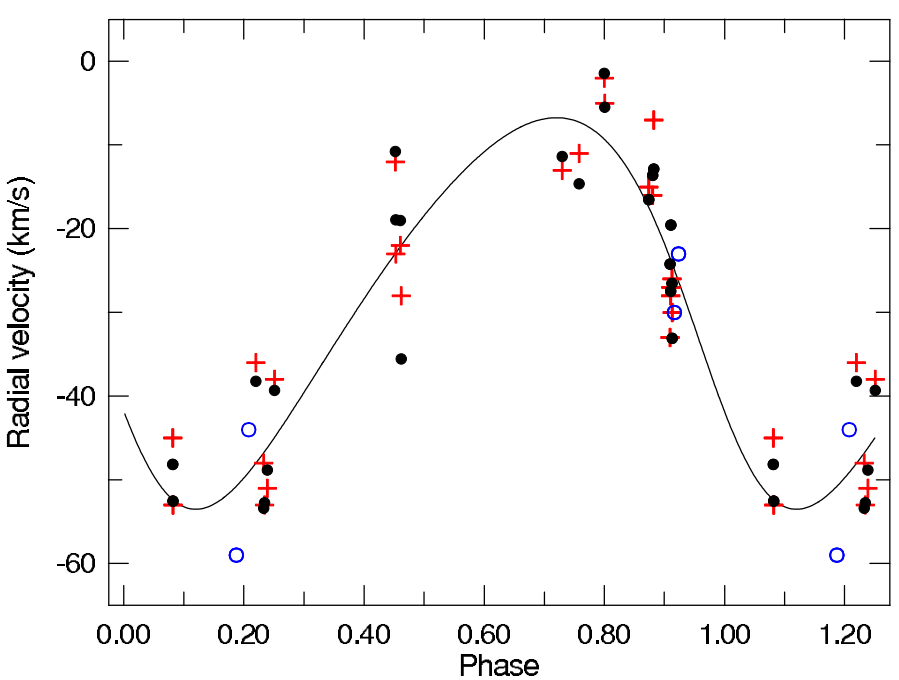

Fig. 10. Long orbit of HD 123056 (binary A). The curve corresponds to elements $P=1314$ days, $e=0.18, K=23 \mathrm{~km} \mathrm{~s}^{-1}, V_{\gamma}=-27 \mathrm{~km} \mathrm{~s}^{-1}$ ). Black full circles indicate measurement of the line He I $5876 \AA$ while red plus symbols represent measurement of the line He II $5411 \AA$ and blue open circles indicate Radcliffe data.

Table 9. RVs based on the Gaussian fits of He I $5876 \AA$ line of the primary component of HD 164438 (in $\mathrm{km} \mathrm{s}^{-1}$ ).

\begin{tabular}{ccrrc}
\hline \hline RJD & Phase & RV with error & O-C & Spg. \\
\hline 54600.8993 & 0.1841 & $6.5 \pm 1.3$ & 0.6 & $\mathrm{~F}$ \\
54937.8665 & 0.0576 & $-19.0 \pm 2.2$ & 0.6 & $\mathrm{~B}$ \\
54943.8670 & 0.6430 & $-2.7 \pm 1.8$ & 1.5 & $\mathrm{~B}$ \\
54976.9109 & 0.8667 & $-33.6 \pm 1.6$ & 0.8 & $\mathrm{~F}$ \\
55072.6480 & 0.2065 & $6.6 \pm 1.8$ & -1.7 & $\mathrm{~B}$ \\
55121.5368 & 0.9759 & $-39.7 \pm 1.8$ & -0.1 & $\mathrm{~B}$ \\
55697.9411 & 0.2082 & $8.0 \pm 1.2$ & -0.8 & $\mathrm{~F}$ \\
55698.8548 & 0.2973 & $13.1 \pm 1.2$ & 0.7 & $\mathrm{~F}$ \\
55798.6818 & 0.0362 & $-27.8 \pm 2.0$ & -1.4 & $\mathrm{~B}$ \\
55812.6264 & 0.3966 & $10.2 \pm 1.8$ & -0.9 & $\mathrm{~B}$ \\
55813.6076 & 0.4923 & $6.4 \pm 1.9$ & -0.4 & $\mathrm{~B}$ \\
55814.6200 & 0.5910 & $-1.0 \pm 1.9$ & -1.0 & $\mathrm{~B}$ \\
55815.6204 & 0.6886 & $-9.7 \pm 1.6$ & -0.3 & $\mathrm{~B}$ \\
55816.6211 & 0.7863 & $-23.3 \pm 1.8$ & -1.4 & $\mathrm{~B}$ \\
55817.6199 & 0.8837 & $-37.3 \pm 1.6$ & -0.9 & $\mathrm{~B}$ \\
55818.6224 & 0.9815 & $-38.3 \pm 1.6$ & 0.6 & $\mathrm{~B}$ \\
55819.6209 & 0.0789 & $-13.0 \pm 1.6$ & 0.8 & $\mathrm{~B}$ \\
55821.6047 & 0.2724 & $11.9 \pm 1.7$ & -0.1 & $\mathrm{~B}$ \\
56067.9290 & 0.3031 & $13.6 \pm 1.3$ & 2.0 & $\mathrm{~F}$ \\
56441.9326 & 0.7898 & $-21.3 \pm 1.7$ & 1.2 & $\mathrm{~B}$ \\
\hline
\end{tabular}

Table 10. New orbital elements for HD 164438; same notation as in Table 3.

\begin{tabular}{lr}
\hline \hline Element & Value \\
\hline$P$ [days] & $10.25042(40)$ \\
$T_{\text {periasrt. }}[\mathrm{RJD}]$ & $55501.05(6)$ \\
$e$ & $0.310(13)$ \\
$\omega\left[^{\circ}\right]$ & $218.5(2.5)$ \\
$K_{1}\left[\mathrm{~km} \mathrm{~s}^{-1}\right]$ & $26.9(4)$ \\
$\gamma_{1}\left[\mathrm{~km} \mathrm{~s}^{-1}\right]$ & $-8.0(3)$ \\
$f(m)\left[M_{\odot}\right]$ & $0.0178(8)$ \\
$v_{1} \sin i\left[\mathrm{~km} \mathrm{~s}^{-1}\right]$ & $89(2)$ \\
$\mathrm{rms}_{1}\left[\mathrm{~km} \mathrm{~s}^{-1}\right]$ & 1.1 \\
\hline \multicolumn{2}{c}{} \\
\hline
\end{tabular}




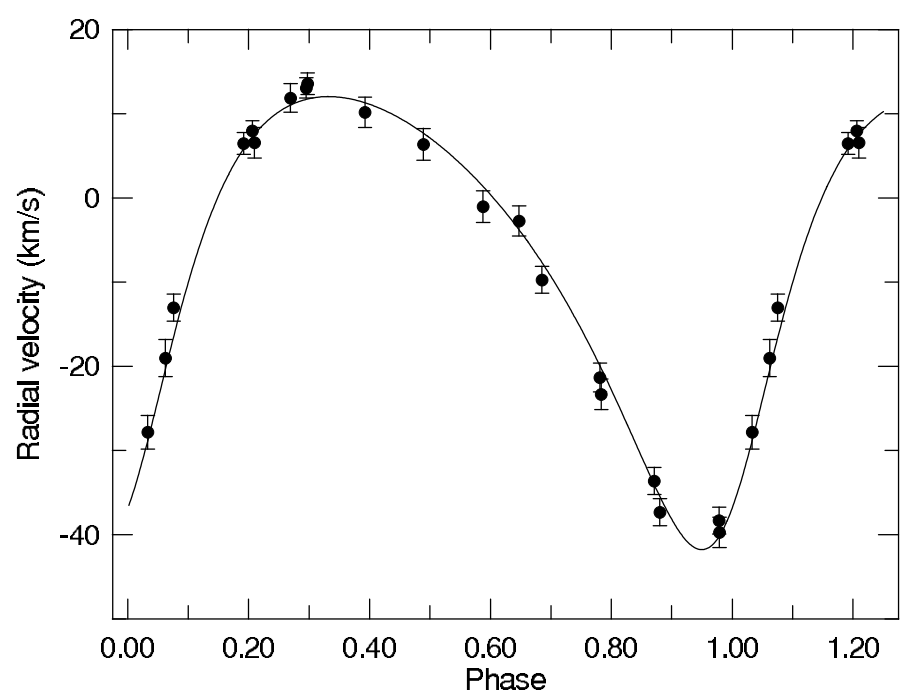

Fig. 11. RV curve of HD 164438 based on Gaussian fits of the He I $5876 \AA$ A line plotted for the ephemeris of Table 10 .

by Drilling \& Perry (1981). Its visual brightness is $V=10$. 09 and its variability was discovered by the HIPPARCos satellite (Perryman \& ESA 1997).

The orbital period and a linear ephemeris

$T_{\text {min.I }}=$ RJD $52096.30+23$ d 446 ,

was derived by Otero (2003) from the HIPPARCos $H_{\mathrm{p}}$ and ASAS $3 V$ photometry.

There are seven FEROS and seven HARPS spectra in the ESO archive (exposed by Dr. Helminiak, with one exception: a FEROS spectrum at RJD 57160 was obtained in the service mode of the "Tycho Brahe" program). These reduced spectra are available as pipeline products. As their phase distribution over the RV curve contained gaps, we also obtained a series of $R \approx 28000$ spectra with the CHIRON spectrograph on the CTIO $1.5 \mathrm{~m}$. All RVs based on the Gaussian fits are listed in Table 11. We also measured RVs using SPEFO, but the results were nearly identical to those derived via Gaussian fitting, so we do not reproduce them here. The profile of the line He I $6678 \AA$ at phase close to elongation is shown in Fig. 12. As V1082 Sco is heavily reddened (e.g. Moffat et al. 1977, $E_{B-V}=1.57$ ), there are numerous diffuse interstellar bands in the spectra. Those around the line He I $6678 \AA$ were also observed in V1182 Aql (Mayer et al. 2005): $6660.5,6672.4$ and $6689.4 \AA$, for example.

Although the measurements for the different He I lines are not identical, the RV curve is well defined and the solution leads to reliable elements. They are listed in Table 12 for the solution based on RVs from the Gaussian fits of the He I $6678 \AA$ line, with weights 1 for the primary, and 0.4 for the secondary RVs. The RVs close to conjunctions were not included in the solution.

A solution of the HIPPARCOS and ASAS-3 light curves has already been obtained by Zasche (2012), but the results had relatively large errors. As the orbital eccentricity and the longitude of periastron are now also better constrained with RVs, our combined light-curve (lc) and RV solution resulted in more accurate elements (Table 13); the fits of the ASAS and HIPPARCos light curves and of our RV curves are shown in Fig. 13 (the RV curve based on the He I $6678 \AA$ line was used since this line is free of emission and represents the true orbital motion better than the He I $5876 \AA$ line). Our improved linear ephemeris is

$T_{\text {min.I }}=$ RJD $53081.05+23.445975$.
The more massive star is larger and cooler, and its spectral lines are considerably more pronounced. When parameters of the components are compared with evolutionary models (e.g. by Claret 2004), it appears that the age of both components is approximately 6 Myr.

We note that when the RV curves are solved independently from the light-curves (see Table 12), slightly different values of the eccentricity and the argument of periastron are obtained: $e=0.287 \pm 0.011$ and $\omega=343.7 \pm 2.6$. This might be the effect of circumbinary matter (CBM), similar to that which causes non-zero eccentricity of RV curves of other early-type binaries (Mayer \& Drechsel 2006). Nevertheless our uncertainty estimates for the combined (light-curve and RV-curve) solution are likely underestimated and the difference insignificant.

In the solution of light curves, one must adopt the effective temperature for one of the components. In this particular case, the primary $T_{\text {eff }}$ is difficult to estimate; for example, the He I lines are almost insensitive to temperature in the expected interval, and their EW, as well as that of other lines, are rather large when compared to synthetic spectra. Fortunately, the effective temperature of the less massive component is relatively well constrained. The He II $5411 \AA$ line is marginally present and the lines of the C II 6578 and $6583 \AA$ doublet are absent. For $\log g \sim 3.25$, this restricts $T_{2, \text { eff }}$ to $29000 \pm 1000 \mathrm{~K}$. The primary temperature, which follows from the PHOEBE solution is then compatible with the expectation for a B0.5/0.7 supergiant (Fraser et al. 2010; Aller et al. 1982; McErlean et al. 1999).

A disturbing fact is that the systemic velocity $\gamma$ of the primary is more negative than that of the secondary by $35 \mathrm{~km} \mathrm{~s}^{-1}$. Such a difference is often present among early-type stars. A very similar result has also been obtained for another supergiant binary V1765 Cyg (Hill \& Fisher 1984; Mayer et al. 1991), although in this latter case, the detection of the secondary lines was uncertain; however, in V1082 Sco the situation is extreme. A strong stellar wind of a binary component is commonly considered as the reason for a blue shift of its $V_{\gamma}$ velocity. It is known that the mass-loss rate by a radiatively driven wind increases in proportion to the size of the star, and is especially enhanced in the case of an evolved supergiant star. This is why the lines of the primary are systematically blue-shifted with respect to those of the secondary.

As the $\mathrm{H} \alpha$ profile shows emission typical for an early supergiant (see Fig. 14), it is clear that some CBM must be present. However, it is not easy to correlate the emission with either the primary or the secondary star. We may note, however, that in some spectra the RV of the emission peak follows the primary's $\mathrm{RV}$, being more positive by $\approx 150 \mathrm{~km} \mathrm{~s}^{-1}$.

In HD 318015, yet another uncommon characteristic is present: the depth of the He I lines of both components change severely, ratio of depths reaches 1:2 and the changes are only partly phase dependent.

The estimated rotational velocities imply rotational periods of 14.1 and 8.0 days for the primary and secondary, respectively. The expected $v \sin i$ for the synchronisation at periastron are $128 \mathrm{~km} \mathrm{~s}^{-1}$ for the primary and $64 \mathrm{~km} \mathrm{~s}^{-1}$ for the secondary. In other words, the primary seems to be synchronised at periastron while the secondary is rotating almost twice as rapidly than what would correspond to synchronisation at periastron.

The parameters of the binary components can be compared with the evolutionary models. We used the models by Claret (2004) and Padova models ${ }^{1}$ (Bressan et al. 2012; Rosenfield et al. 2016) for masses of 20 to $30 M_{\odot}$. The Claret

\footnotetext{
Available at http://stev . oapd.inaf.it/cgi-bin/cmd
} 
Table 11. RVs based on the Gaussian fits of He I line profiles of HD 318015 (in km s${ }^{-1}$ ).

\begin{tabular}{|c|c|c|c|c|c|c|c|c|c|c|c|c|}
\hline \multirow[t]{2}{*}{ RJD } & \multirow[t]{2}{*}{ Phase } & \multicolumn{3}{|c|}{6678 primary } & \multicolumn{3}{|c|}{6678 secondary } & \multicolumn{2}{|c|}{5876 primary } & \multicolumn{2}{|c|}{5876 secondary } & \multirow[t]{2}{*}{ Spg. } \\
\hline & & RV & error & $\mathrm{O}-\mathrm{C}$ & RV & error & $\mathrm{O}-\mathrm{C}$ & & error & & error & \\
\hline 55721.7233 & 0.5107 & -120 & 3 & -4.1 & 102 & 5 & -1.7 & -130 & 3 & 107 & 8 & $\mathrm{H}$ \\
\hline 55722.6926 & 0.5520 & -124 & 3 & -3.0 & 104 & 5 & -5.6 & -131 & 3 & 108 & 8 & $\mathrm{H}$ \\
\hline 56100.6215 & 0.6712 & -116 & 4 & 1.3 & 97 & 8 & -8.3 & -139 & 4 & 111 & 7 & $\mathrm{~F}$ \\
\hline 56137.5210 & 0.2450 & - & - & - & - & & - & -45 & & -19 & & $\mathrm{H}$ \\
\hline 56137.7109 & 0.2531 & - & - & - & - & & _- & -41 & & -23 & & $\mathrm{H}$ \\
\hline 56138.5066 & 0.2879 & - & - & - & - & & - & -41 & & -35 & & $\mathrm{H}$ \\
\hline 56138.6052 & 0.2912 & - & - & - & - & & - & -41 & & -35 & & $\mathrm{H}$ \\
\hline 56179.5219 & 0.0364 & 134 & 4 & 2.3 & -179 & 3 & 2.5 & 129 & 3 & -176 & 4 & $\mathrm{H}$ \\
\hline 56193.6246 & 0.6379 & -124 & 4 & -2.6 & 99 & 8 & -11.1 & -131 & 5 & 114 & 9 & $\mathrm{~F}$ \\
\hline 56376.8520 & 0.4580 & -92 & 5 & 11.9 & 85 & 10 & -4.9 & -97 & 5 & 107 & 9 & $\mathrm{~F}$ \\
\hline 56428.9173 & 0.6734 & -108 & 5 & 8.9 & 98 & 8 & -6.8 & -107 & 4 & 126 & 7 & $\mathrm{~F}$ \\
\hline 56517.5751 & 0.4548 & -97 & 4 & 7.4 & 98 & 7 & 7.5 & -106 & 3 & 126 & 7 & $\mathrm{~F}$ \\
\hline 56520.7121 & 0.5886 & -120 & 4 & 2.0 & 119 & 7 & 7.1 & -125 & 3 & 141 & 3 & $\mathrm{~F}$ \\
\hline 57131.7581 & 0.6505 & -115 & 3 & 5.2 & 106 & 4 & -2.6 & -116 & 3 & 121 & 4 & $\mathrm{C}$ \\
\hline 57132.7388 & 0.6923 & -106 & 3 & 7.1 & 95 & 5 & -5.5 & -113 & 4 & 100 & 9 & $\mathrm{C}$ \\
\hline 57133.7728 & 0.7364 & -95 & 6 & 5.0 & 88 & 10 & 2.7 & -98 & 4 & 97 & 8 & $\mathrm{C}$ \\
\hline 57134.8617 & 0.7828 & -77 & 7 & 1.2 & 82 & 13 & 21.7 & -82 & 6 & 59 & 11 & $\mathrm{C}$ \\
\hline 57135.8232 & 0.8238 & -42 & 7 & 8.7 & 41 & 13 & 12.4 & -51 & 6 & 31 & 12 & $\mathrm{C}$ \\
\hline 57136.6796 & 0.8604 & - & - & - & - & - & - & - & - & -69 & - & $\mathrm{C}$ \\
\hline 57139.7835 & 0.9927 & 121 & 3 & 2.4 & -175 & 6 & -8.6 & 114 & 3 & -161 & 4 & $\mathrm{C}$ \\
\hline 57143.7792 & 0.1632 & 52 & 6 & -1.7 & -94 & 11 & -2.4 & 42 & 6 & -107 & 10 & $\mathrm{C}$ \\
\hline 57143.8172 & 0.1648 & 52 & 6 & -0.3 & -93 & 11 & -2.9 & 40 & 6 & -103 & 10 & $\mathrm{C}$ \\
\hline 57145.8735 & 0.2525 & - & - & - & - & - & - & -36 & & -4 & - & $\mathrm{C}$ \\
\hline 57146.7851 & 0.2914 & - & - & - & - & _- & - & -46 & & -6 & - & $\mathrm{C}$ \\
\hline 57148.7816 & 0.3765 & -96 & 5 & -16.7 & - & - & - & -99 & 9 & -20 & 11 & $\mathrm{C}$ \\
\hline 57150.7244 & 0.4594 & -115 & 3 & -9.4 & 93 & 5 & 1.2 & -122 & 4 & 95 & 5 & $\mathrm{C}$ \\
\hline 57151.7681 & 0.5039 & -124 & 3 & -9.2 & 97 & 4 & -5.5 & -137 & 3 & 106 & 4 & $\mathrm{C}$ \\
\hline 57152.8382 & 0.5495 & -127 & 3 & -6.2 & 106 & 3 & -3.4 & -141 & 4 & 129 & 5 & $\mathrm{C}$ \\
\hline 57154.7004 & 0.6290 & -125 & 3 & -2.9 & 103 & 3 & -7.9 & -131 & 4 & 104 & 12 & $\mathrm{C}$ \\
\hline 57155.7111 & 0.6721 & -125 & 3 & -7.9 & 103 & 4 & -2.1 & -128 & 4 & 105 & 12 & $\mathrm{C}$ \\
\hline 57156.7256 & 0.7153 & -102 & 3 & 5.0 & 100 & 4 & 6.5 & -108 & 4 & 105 & 6 & $\mathrm{C}$ \\
\hline 57157.7168 & 0.7576 & -86 & 5 & 5.1 & 80 & 7 & 4.8 & -85 & 5 & 92 & 9 & $\mathrm{C}$ \\
\hline 57158.7443 & 0.8014 & -66 & 7 & 0.8 & 64 & 15 & 16.9 & -71 & 6 & 35 & 12 & $\mathrm{C}$ \\
\hline 57160.8764 & 0.8924 & - & - & - & - & - & - & - & - & 13 & - & $\mathrm{T}$ \\
\hline 57161.7600 & 0.9301 & 42 & 7 & -16.3 & -109 & 13 & -12.1 & 34 & 6 & -125 & 11 & $\mathrm{C}$ \\
\hline 57163.6970 & 0.0127 & 133 & 4 & 4.7 & -172 & 5 & 5.6 & 132 & 3 & -164 & 4 & $\mathrm{C}$ \\
\hline 57164.6187 & 0.0520 & 133 & 5 & 3.7 & -172 & 7 & 6.8 & 132 & 3 & -164 & 5 & $\mathrm{C}$ \\
\hline 57165.6736 & 0.0970 & 107 & 3 & 0.4 & -162 & 4 & -19.4 & 101 & 3 & -187 & 6 & $\mathrm{C}$ \\
\hline 57166.6550 & 0.1388 & 71 & 4 & -3.0 & -100 & 7 & 15.0 & 69 & 4 & -87 & 8 & $\mathrm{C}$ \\
\hline
\end{tabular}

models are shown in Fig. 15; the fit is poor, and is not improved when the Padova models are used. Although the mass of the donor is commonly accepted as higher, in this case both components are over-luminous for their masses. In order to make the agreement better, the components would have to be more massive, larger, or cooler. As the masses and radii appear as well determined, the temperature might be suspected, although the necessary difference of $\approx 4000 \mathrm{~K}$ is not compatible with the characteristics of the spectra.

We note that comparisons of OB supergiant spectra with line-blanketed model atmospheres and determination of their physical properties was carried out by Crowther et al. (2006) and Fraser et al. (2010), for example. In Fig. 16, the properties of the supergiants studied by Crowther et al. (2006) and those of the components of V1082 Sco are compared with the evolutionary tracks by Schaerer et al. (1993), Schaller et al. (1993). The primary component fits the line defined by the other spectra perfectly; note, however, that the stars shown there have masses from 14 to $46 M_{\odot}$ and do not indicate any obvious dependence of the mass on the effective temperature. The spectra studied by Fraser et al. (2010) indicate a similar behaviour; see their Fig. 3. Although they occupy a correct area, the fit with masses does not exist.

We underline that in the above exercises, we deliberately used several different grids of stellar evolutionary models to demonstrate that the discrepancy is inherent to the systems and does not depend on the choice of a particular set of evolutionary models. Using common procedures, the distance of the binary appears to be $2800 \mathrm{pc}$. The binary is a member of the open cluster Trumpler 27. Although there are doubts about the reality of the cluster, several similar early-type stars in the vicinity of V1082 Sco are certain (Moffat et al. 1977).

In passing we note that the only eclipsing binary in our sample, V1082 Sco, can be compared to V1176 Cen, eclipses of which were discovered by Otero \& Wils (2005), who used the $V$ observations from the ASAS3 survey; the period of V1176 Cen 


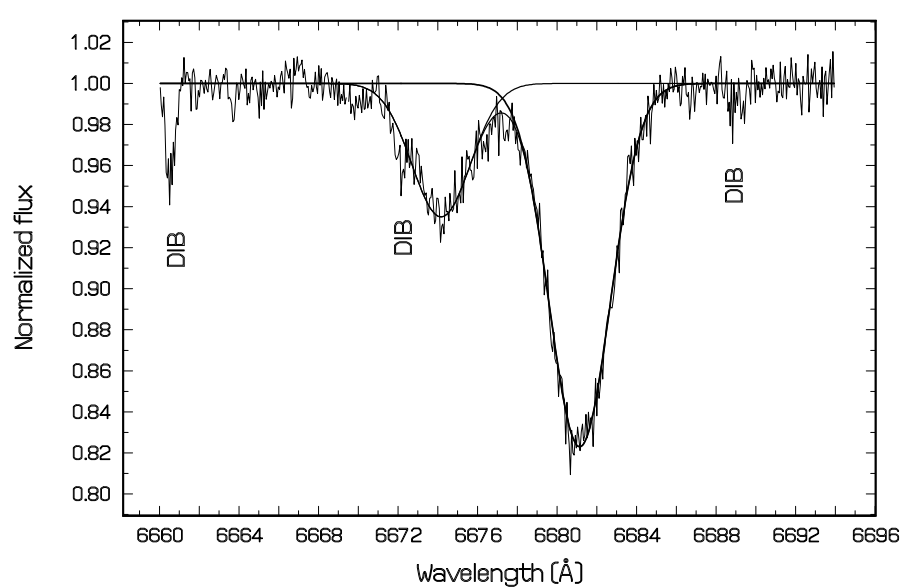

Fig. 12. An example of He I 6678 line profile at phase near elongation of HD 318015 .

Table 12. Preliminary orbital elements for HD 318015 based solely on the RVs from the Gaussian fits of the He I $6678 \AA$ line; same notation as in Table 3 .

\begin{tabular}{lr}
\hline \hline Element & Value \\
\hline$P$ [days] & 23.44598 fixed \\
$T_{\text {periastr. }}[\mathrm{RJD}]$ & $52075.91(15)$ \\
$e$ & $0.287(11)$ \\
$\omega\left[{ }^{\circ}\right]$ & $343.7(2.6)$ \\
$K_{1}\left[\mathrm{~km} \mathrm{~s}^{-1}\right]$ & $128.61(37)$ \\
$K_{2}\left[\mathrm{~km} \mathrm{~s}^{-1}\right]$ & $147.47(40)$ \\
$\gamma_{1}\left[\mathrm{~km} \mathrm{~s}^{-1}\right]$ & $-28.8(1.3)$ \\
$\gamma_{2}\left[\mathrm{~km} \mathrm{~s}^{-1}\right]$ & $4.0(1.8)$ \\
$m_{1} \sin ^{3} i\left[M_{\odot}\right]$ & $24.0(2)$ \\
$m_{2} \sin ^{3} i\left[M_{\odot}\right]$ & $20.9(2)$ \\
$a \sin i\left[R_{\odot}\right]$ & $122.6(9)$ \\
$v_{1} \sin i\left[\mathrm{~km} \mathrm{~s}^{-1}\right]$ & $119(5)$ \\
$v_{2} \sin i\left[\mathrm{~km} \mathrm{~s}^{-1}\right]$ & $104(8)$ \\
$\mathrm{rms}_{1}\left[\mathrm{~km} \mathrm{~s}^{-1}\right]$ & 7.0 \\
$\mathrm{rms}_{2}\left[\mathrm{~km} \mathrm{~s}^{-1}\right]$ & 7.0 \\
\hline
\end{tabular}

is 31.029. Solving this ASAS3 light curve with PHOEBE, we found relative radii of 0.154 and 0.0724 , and an inclination of $87^{\circ} 9$. If $T_{1}=22000 \mathrm{~K}$ is assumed, then $T_{2}=17600 \mathrm{~K}$.

\section{Conclusions}

Studying seven high-mass objects, we found that a detailed investigation has increased the multiplicity status of each system, be it from single to binary or from binary to triple. Table 14 shows that the secondary components are $\mathrm{O}$ and $\mathrm{B}$-type stars. In at least two cases, the tertiary component is also a massive star. Although the sample is small, the number of multiple systems is surprisingly high. Five out of seven systems studied here contain a third component or are suspected to have one.

The two short-period $P_{\text {inner }}<7$ d systems (HD $92206 \mathrm{C}$ and HD 123056) were both identified as (at least) triple systems in agreement with statistical analysis by Tokovinin et al. (2006), who reported that $96 \%$ of binaries with a period of less than three days are at least triple systems. The multiplicity fraction among the remaining long-periodic $\left(P_{\text {inner }}>\right.$ $7 \mathrm{~d}$ ) binaries of $\approx 50 \%$ also agrees with their findings. It is difficult to discuss the formation process of the individual multiples, because with the exception of HD 123056, the
Table 13. Combined PHOEBE solution of the light and RV curves of HD 318015 .

\begin{tabular}{|c|c|}
\hline Element & Value \\
\hline Period $P[\mathrm{~d}]$ & $23.445975(78)$ \\
\hline$T_{\text {periastr. }}[\mathrm{RJD}]$ & $53083.80(16)$ \\
\hline$T_{\operatorname{min.I}}[\mathrm{RJD}]$ & 53081.05 \\
\hline$T_{\text {min.II }}[\mathrm{RJD}]$ & 53065.65 \\
\hline Mass ratio $q$ & $0.861(5)$ \\
\hline Semimajor axis $a\left[R_{\odot}\right]$ & $123.7(1.2)$ \\
\hline Eccentricity $e$ & $0.2675(12)$ \\
\hline Argument of periastron $\omega\left[^{\circ}\right]$ & $338.3(1.0)$ \\
\hline Fract. radius $r_{1}$ [pole] & $0.2613(21)$ \\
\hline Fract. radius $r_{2}$ [pole] & $0.1371(11)$ \\
\hline Inclination [deg] & $93.9(0.7)$ \\
\hline Radius $R_{1}\left[R_{\odot}\right]$ & $33.39(55)$ \\
\hline Radius $R_{2}\left[R_{\odot}\right]$ & $17.05(40)$ \\
\hline$T_{1}[\mathrm{~K}]$ & $24940(590)$ \\
\hline$T_{2}[\mathrm{~K}]$ & $29000(1000)$ \\
\hline$m_{1}\left[M_{\odot}\right]$ & $24.80(38)$ \\
\hline$m_{2}\left[M_{\odot}\right]$ & $21.36(35)$ \\
\hline$L_{1}[V]$ & $0.7490(33)$ \\
\hline$L_{1}\left[H_{\mathrm{p}}\right]$ & $0.7447(32)$ \\
\hline$L_{2} / L_{1}$ & $0.3427(62)$ \\
\hline$M_{1}^{\text {bol }}[\mathrm{mag}]$. & $-9.23(1)$ \\
\hline$M_{2}^{\text {bol }}$ [mag.] & $-8.43(2)$ \\
\hline $\log g_{1}[\mathrm{cgs}]$ & $2.785(16)$ \\
\hline $\log g_{2}[\mathrm{cgs}]$ & $3.304(14)$ \\
\hline Synchronicity ratio $F_{1}$ & 1.00 (assumed) \\
\hline Synchronicity ratio $F_{2}$ & 1.00 (assumed) \\
\hline
\end{tabular}

Notes. Error estimates are based on repeated runs of PHOEBE with a different secondary effective temperature kept fixed throughout the fitting. All runs together uniformly sample the interval $T_{2} \in[28000 ; 30000] \mathrm{K}$.

properties of the outer orbit are unknown. Nonetheless, the members of the inner binaries of both short-period systems have close-to-equal masses, which is a typical outcome of accretion from a circumbinary disk (Bate et al. 2002). The inner and outer orbits of HD 123056 are likely close enough for the Kozai-cycle with tides (Eggleton \& Kiseleva-Eggleton 2001; Fabrycky \& Tremaine 2007) to play some role in the shrinkage of its inner orbit. However, without knowing the orbital elements of both orbits, it is impossible to (dis)prove such a conjecture.

Our results for $v \sin i$ values seem to confirm the conclusion of Ramírez-Agudelo et al. (2015) about the lack of very high rotational velocities among O-type binary systems, at least for cases where we have some idea about the orbital inclinations.

Our general conclusion is that the ultimate decision among various theories of the massive-star origin might only come when the binary properties of a representative sample of systems, based on individual orbital solutions, and the light-curve solutions in case of eclipsing (sub)systems, are known. So far, the knowledge of duplicity and/or multiplicity of OB stars has mainly been deduced using statistics.

Furthermore, we note that the rapidly maturing optical interferometry can also significantly contribute to achieving the above-mentioned goal. For instance, the primary of HD $93146 \mathrm{~A}$ is the earliest star in our sample and has a long period of $1131.4 \mathrm{~d}$. Both this star and HD 123056, another star that probably has a period of $1314 \mathrm{~d}$, might be suitable targets for interferometry. HD 123056 was already observed by Aldoretta et al. (2015), but not separated; higher resolution than 10 mas is 

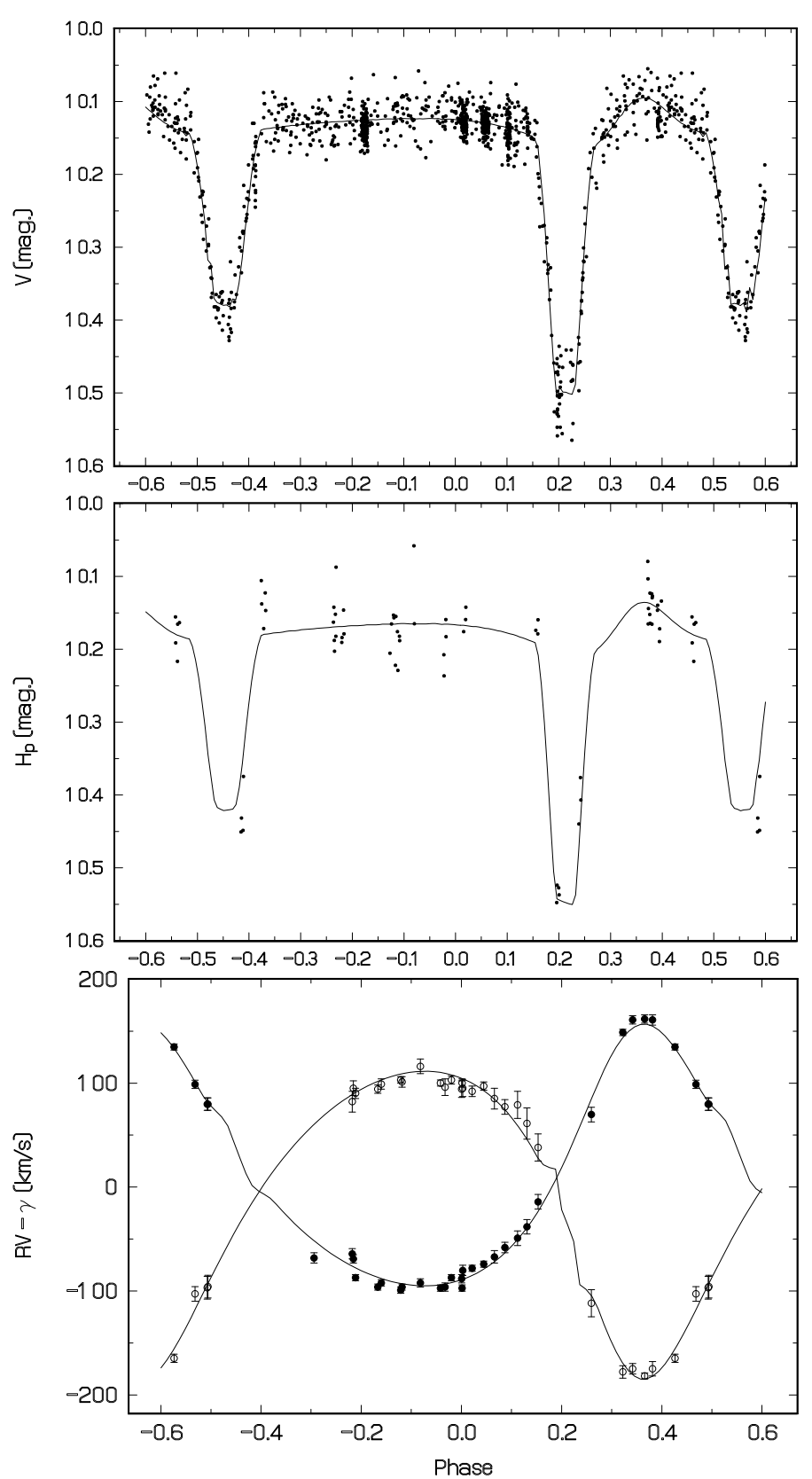

Fig. 13. ASAS3 $V$ and Hipparcos $H_{\mathrm{p}}$ light curves and the orbital RV curve of HD 318015 based on RVs from the Gaussian fits of the He I $6678 \AA$ Aline. Phase zero corresponds to RJD 53076.08 , chosen as an arbitrary reference epoch in PHOEBE. The phases of the primary and secondary minima according to the combined PHOEBE solution are then 0.2120 and -0.4449 , respectively.

probably needed. Also in the case of HD 93146 A, high resolution is necessary; at a distance of $\approx 3.0 \mathrm{kpc}$ its semi-major axis might be of the order of 2.5 mas.

Acknowledgements. This publication is supported as a project of the NordrheinWestfälische Akademie der Wissenschaften und der Künste in the framework of the academy programme by the Federal Republic of Germany and the state Nordrhein-Westfalen. In the early stages of this project, the research of P.H., P.M., and J.N. was supported by the grant P209/10/0715 of the Czech Science Foundation. J.N. and P.H. were also supported from the grant GA15-02112S of the Czech Science Foundation and from the grant No. 250015 of the Grant Agency of the Charles University in Prague. We are grateful for the help of A. Barr Domínguez, K. Fuhrmann, L. Kaderhandt and M. Ramolla during the observations and the reduction. We also thank High Point University for providing the funds necessary to purchase observing time on the CTIO $1.5 \mathrm{~m}$ telescope

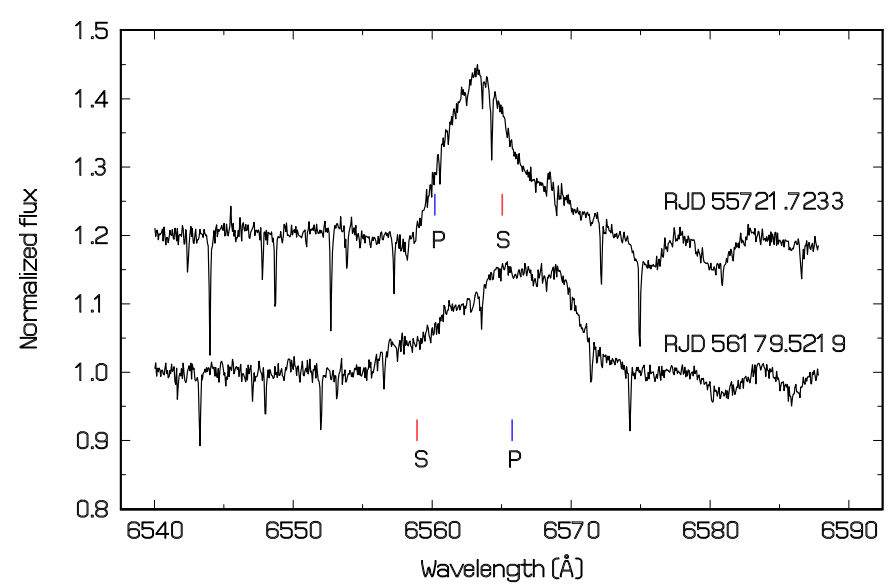

Fig. 14. Two examples of $\mathrm{H} \alpha$ line profiles of HD 318015. The $\mathrm{C}$ II doublet at 6578 and $6582 \AA$ is also seen. The vertical dashes show the position of the primary (blue) and the secondary (red) in both $\mathrm{H} \alpha$ profiles.

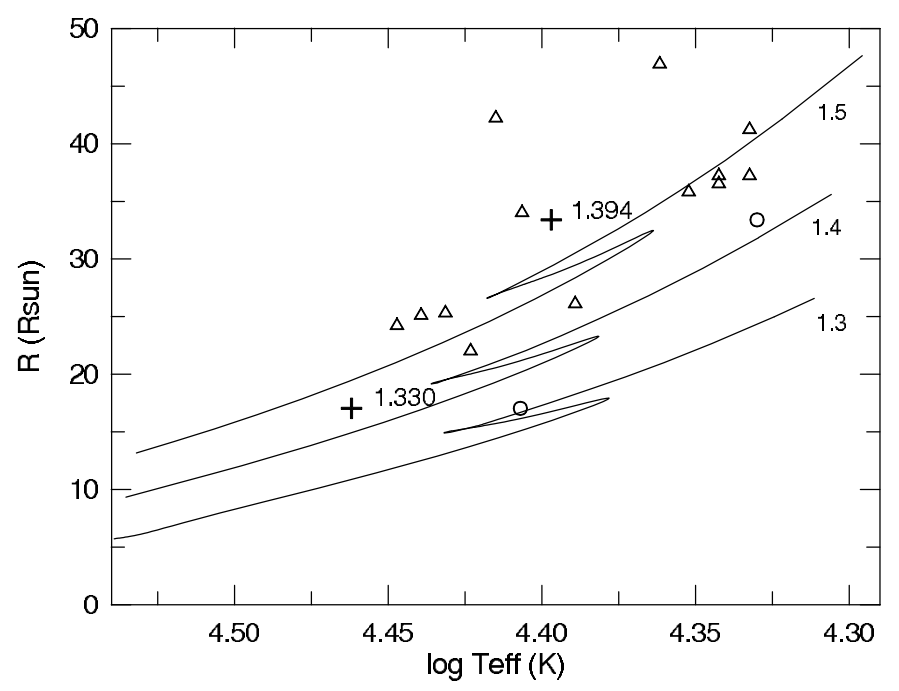

Fig. 15. HD 318015: the lines show the relation between $\log T_{\text {eff }}$ and $R$ for the evolutionary models published by Claret (2004). The lines are labelled by $\log M$. Two plus symbols are the positions of both binary components, also labelled by $\log M$. Two circles show where the stars with the given masses and radii should be located.

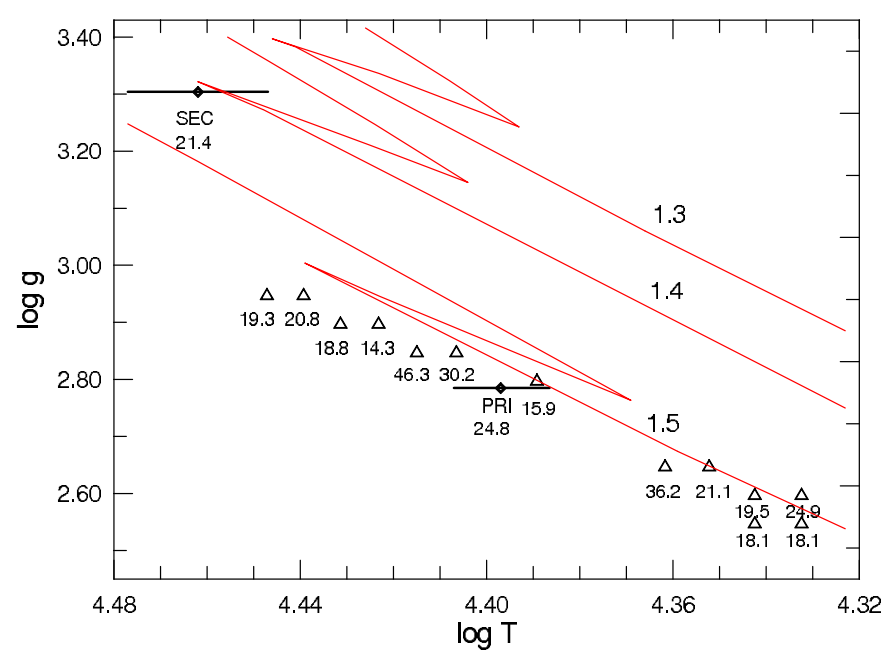

Fig. 16. A comparison of the properties of the components of V1082 Sco with those for OB supergiants studied by Crowther et al. (2006) in the $\log g$ vs. $\log T_{\text {eff }}$ diagram. Evolutionary tracks from Schaerer et al. (1993) are also shown. Note that there is no clear correspondence between the $T_{\text {eff }}$ and stellar masses. 
Table 14. Multiplicity properties of the new OB systems.

\begin{tabular}{ccccc}
\hline \hline HD & Primary & Secondary & $m_{2} / m_{1}$ & Tertiary \\
\hline $92206 \mathrm{C}$ & O8 V & O9.7 V & 0.75 & B2: \\
$93146 \mathrm{~A}$ & O6.5 V & B0.5 & 0.48 & O9.7 IV $^{1}$ \\
112244 & O8.5 Iab & late B? & $0.06-0.12$ & unknown \\
123056 & O8.5 & B0: & 0.70 & B0: \\
164438 & O9.2 IV & later B3 & $0.10-0.22$ & probable \\
164816 & O9.5 V & B0 V & 0.90 & yes \\
318015 & B0.7 Ia & O9.5 III & 0.81 & unknown \\
\hline
\end{tabular}

Notes. Spectral types marked by a colon (:) are uncertain. ${ }^{(1)}$ The physical association of this nearby star needs to be verified.

through the SMARTS Consortium, and for funding part of this work during the 2015 HPU Summer Research Program in the Sciences (SuRPS). The observations obtained with the MPG $2.2 \mathrm{~m}$ telescope were supported by the Czech Ministry of Education, Youth and Sports project LG14013 ("Tycho Brahe: supporting Ground-based Astronomical Observations") during run P2 in May 2015 We would like to thank the observers Drs. P. Kabath and S. Vennes for obtaining the data. We thank Universidad Católica del Norte in Antofagasta, Chile, for continuous support. Our thanks are also due to Dr. M. Hackstein, who put at our disposal the individual photometric observations of HD 92206C. The use of the NASA/ADS bibliographical service and SIMBAD electronic database are gratefully acknowledged.

\section{References}

Aldoretta, E. J., Caballero-Nieves, S. M., Gies, D. R., et al. 2015, AJ, 149, 26 Aller, L. H., Appenzeller, I., Baschek, B., et al., eds. 1982, Landolt-Börnstein: Numerical Data and Functional Relationships in Science and Technology - New Series Gruppe/Group 6 Astronomy and Astrophysics" Volume 2 Schaifers/Voigt: Astronomy and Astrophysics/Astronomie und Astrophysik Stars and Star Clusters/Sterne und Sternhaufen (Springer Verlag)

Barbá, R. H., Gamen, R., Arias, J. I., et al. 2010, Rev. Mex. Astron. Astrofis. Conf. Ser., 38, 30

Bate, M. R., Bonnell, I. A., \& Bromm, V. 2002, MNRAS, 336, 705

Bressan, A., Marigo, P., Girardi, L., et al. 2012, MNRAS, 427, 127

Campillay, A., Arias, J., Barba, R., et al. 2007, in VI Reunion Anual Sociedad Chilena de Astronomia (SOCHIAS), 63

Chini, R., Hoffmeister, V. H., Nasseri, A., Stahl, O., \& Zinnecker, H. 2012, MNRAS, 424, 1925

Claret, A. 2004, A\&A, 424, 919

Crowther, P. A., Lennon, D. J., \& Walborn, N. R. 2006, A\&A, 446, 279

Desmet, M., Frémat, Y., Baudin, F., et al. 2010, MNRAS, 401, 418

Drilling, J. S., \& Perry, C. L. 1981, A\&AS, 45, 439

Eggleton, P. P., \& Kiseleva-Eggleton, L. 2001, ApJ, 562, 1012

Fabrycky, D., \& Tremaine, S. 2007, ApJ, 669, 1298

Feast, M. W., Thackeray, A. D., \& Wesselink, A. J. 1963, MmRAS, 68,

Fernandes, B., Gregorio-Hetem, J., \& Hetem, A. 2012, A\&A, 541, A95

Fraser, M., Dufton, P. L., Hunter, I., \& Ryans, R. S. I. 2010, MNRAS, 404, 1306

Fuhrmann, K., Chini, R., Hoffmeister, V. H., et al. 2011, MNRAS, 411, 2311

Gaia Collaboration (Brown, A. G. A., et al.) 2016, A\&A, 595, A2

Garmany, C. D., Conti, P. S., \& Massey, P. 1980, ApJ, 242, 1063

Hackstein, M., Fein, C., Haas, M., et al. 2015, Astron. Nachr., 336, 590

Hadrava, P. 2004a, Publ. Astron. Inst. Acad. Sci. Czech Rep., 92, 1

Hadrava, P. 2004b, Publ. Astron. Inst. Acad. Sci. Czech Rep., 92, 15

Harmanec, P. 1989, Bull. Astron. Inst. Czechoslov., 40, 201

Harmanec, P. 1999, A\&A, 341, 867

Harmanec, P., Holmgren, D. E., Wolf, M., et al. 2014, A\&A, 563, A120

Harmanec, P., Koubský, P., Nemravová, J. A., et al. 2015, A\&A, 573, A107

Hill, G., \& Fisher, W. A. 1984, A\&A, 139, 123

Horn, J., Kubát, J., Harmanec, P., et al. 1996, A\&A, 309, 521

Jakate, S. M. 1979, AJ, 84, 552

Kaufer, A., Wolf, B., Andersen, J., \& Pasquini, L. 1997, The Messenger, 89, 1

Kharchenko, N. V., Piskunov, A. E., Röser, S., Schilbach, E., \& Scholz, R.-D. 2005, A\&A, 438, 1163
Kiminki, D. C., \& Kobulnicky, H. A. 2012, ApJ, 751, 4

Kiminki, D. C., Kobulnicky, H. A., Kinemuchi, K., et al. 2007, ApJ, 664, 1102 Kobulnicky, H. A., Kiminki, D. C., Lundquist, M. J., et al. 2014, ApJS, 213, 34 Koen, C., \& Eyer, L. 2002, MNRAS, 331, 45

Levato, H., \& Malaroda, S. 1981, PASP, 93, 714

Levato, H., Malaroda, S., Garcia, B., Morrell, N., \& Solivella, G. 1990, ApJS, 72,323

Levenhagen, R. S., \& Leister, N. V. 2006, MNRAS, 371, 252

Maíz Apellániz, J., Sota, A., Arias, J. I., et al. 2016, ApJS, 224, 4

Marchenko, S. V., Moffat, A. F. J., van der Hucht, K. A., et al. 1998, A\&A, 331, 1022

Martins, F., Schaerer, D., \& Hillier, D. J. 2005, A\&A, 436, 1049

Martins, F., Marcolino, W., Hillier, D. J., Donati, J.-F., \& Bouret, J.-C. 2015, A\&A, 574, A142

Mason, B. D., Gies, D. R., Hartkopf, W. I., et al. 1998, AJ, 115, 821

Mason, B. D., Wycoff, G. L., Hartkopf, W. I., Douglass, G. G., \& Worley, C. E. 2001, AJ, 122, 3466

Mason, B. D., Hartkopf, W. I., Gies, D. R., Henry, T. J., \& Helsel, J. W. 2009, AJ, 137, 3358

Massey, P., DeGioia-Eastwood, K., \& Waterhouse, E. 2001, AJ, 121, 1050

Mayer, P., \& Drechsel, H. 2006, The Observatory, 126, 355

Mayer, P., Hadrava, P., Harmanec, P., \& Chochol, D. 1991, Bull. Astron. Inst. Czechoslov., 42, 230

Mayer, P., Drechsel, H., \& Lorenz, R. 2005, ApJS, 161, 171

Mayer, P., Harmanec, P., Sana, H., \& Le Bouquin, J.-B. 2014, AJ, 148, 114

Mayor, M., Pepe, F., Queloz, D., et al. 2003, The Messenger, 114, 20

McErlean, N. D., Lennon, D. J., \& Dufton, P. L. 1999, A\&A, 349, 553

Mermilliod, J. C. 1986, Bulletin d'Information du Centre de Donnees Stellaires, 31, 175

Moffat, A. F. J., Fitzgerald, M. P., \& Jackson, P. D. 1977, ApJ, 215, 106

Munari, U., \& Tomasella, L. 1999, A\&A, 343, 806

Nasseri, A., Chini, R., Harmanec, P., et al. 2014, A\&A, 568, A94

Otero, S. A. 2003, Inf. Bull. Var. Stars, 5480, 1

Otero, S. A., \& Wils, P. 2005, Inf. Bull. Var. Stars, 5644, 1

Owocki, S. P., Cranmer, S. R., \& Fullerton, A. W. 1995, ApJ, 453, L37

Perryman, M. A. C., \& ESA 1997, The HiPPARCOS and TYCHO catalogues, Astrometric and photometric star catalogues derived from the ESA HIPPARcos Space Astrometry Mission (Noordwijk, The Netherlands: ESA Publications Division), ESA SP Series, 1200

Prisinzano, L., Damiani, F., Micela, G., \& Sciortino, S. 2005, A\&A, 430, 941

Prša, A., \& Zwitter, T. 2005, ApJ, 628, 426

Prša, A., \& Zwitter, T. 2006, Ap\&SS, 304, 347

Ramírez-Agudelo, O. H., Sana, H., de Mink, S. E., et al. 2015, A\&A, 580, A92

Rosenfield, P., Marigo, P., Girardi, L., et al. 2016, ApJ, 822, 73

Sana, H., \& Evans, C. J. 2011, in Active OB Stars: Structure, Evolution, Mass Loss, and Critical Limits, eds. C. Neiner, G. Wade, G. Meynet, \& G. Peters, IAU Symp., 272, 474

Sana, H., Gosset, E., Nazé, Y., Rauw, G., \& Linder, N. 2008, MNRAS, 386, 447 Sana, H., James, G., \& Gosset, E. 2011, MNRAS, 416, 817

Sana, H., de Mink, S. E., de Koter, A., et al. 2012, Science, 337, 444

Sana, H., de Koter, A., de Mink, S. E., et al. 2013, A\&A, 550, A107

Sana, H., Le Bouquin, J.-B., Lacour, S., et al. 2014, ApJS, 215, 15

Schaerer, D., Charbonnel, C., Meynet, G., Maeder, A., \& Schaller, G. 1993, A\&AS, 102, 339

Schaller, G., Schaerer, D., Meynet, G., \& Maeder, A. 1993, VizieR Online Data Catalog: IV/09

Škoda, P. 1996, in Astronomical Data Analysis Software and Systems V, ASP Conf. Ser., 101, 187

Sota, A., Maíz Apellániz, J., Morrell, N. I., et al. 2014, ApJS, 211, 10

Stellingwerf, R. F. 1978, ApJ, 224, 953

Stickland, D. J., \& Lloyd, C. 2001, The Observatory, 121, 1

Thackeray, A. D., Tritton, S. B., \& Walker, E. N. 1973, MmRAS, 77, 199

Tokovinin, A., Thomas, S., Sterzik, M., \& Udry, S. 2006, A\&A, 450, 681

Tokovinin, A., Fischer, D. A., Bonati, M., et al. 2013, PASP, 125, 1336

Trepl, L., Hambaryan, V. V., Pribulla, T., et al. 2012, MNRAS, 427, 1014

Walborn, N. R. 1973, AJ, 78, 1067

Walborn, N. R., Nichols-Bohlin, J., \& Panek, R. J. 1985, NASA Reference Publication, 1155

Zasche, P. 2012, Acta Astron., 62, 97

Zinnecker, H., \& Yorke, H. W. 2007, ARA\&A, 45, 481

Zucker, S. 2003, MNRAS, 342, 1291

Zucker, S., \& Mazeh, T. 1994, ApJ, 420, 806

Zucker, S., Torres, G., \& Mazeh, T. 1995, ApJ, 452, 863 